\title{
On internal fluid dynamics
}

\author{
Frank T. Smith
}

Received: 6 September 2011 / Revised: 14 February 2012 / Accepted: 15 February 2012 /

Published online: 21 March 2012

(C) The Author(s) 2012. This article is published with open access at SpringerLink.com

\begin{abstract}
Motivated by a range of applications (biomedical, industrial, engineering, environmental) this contribution is focussed on a mathematical study of (a) constriction/distortion and (b) branching in a vessel or network of vessels containing fluid flow. The central interest addressed is in medium-to-high Reynolds numbers where asymptotic approaches and matching yield much insight. The main reasoning, order arguments and scaling factors within various parts of the vessels are presented. Theory and corresponding analysis are described for aspect (a) in symmetric and nonsymmetric cases and aspect (b) over short or long length scales with or without viscous-inviscid interactions, where attention is given to side-branching, large networks, viscous wall layers, flow reversal, eddies and upstream influence. Three-dimensional effects for (a) and (b) are also investigated. A final discussion includes suggestions of future project topics.
\end{abstract}

Keywords Mathematical modelling - Range of applications · Constriction · Branching $\cdot$ Networks

\section{Introduction}

Internal fluid dynamics associated with the flow through a rigid vessel or network of vessels is described theoretically in the present article which is concentrated in particular on two fundamental aspects, namely (a) constriction and (b) branching of the

Communicated by S.K. Jain.

F. T. Smith (凶)

Department of Mathematics, UCL, Gower Street, London WC1E 6BT, UK

e-mail: frank@math.ucl.ac.uk 
containing vessels. These aspects are of special interest in practical [1-15] as well as theoretical terms [16-30].

The practical interest lies in numerous industrial and engineering related contexts but especially in biomedical applications, which in a sense motivate the current studies. In arteries within the human cardiovasculature and likewise in the respiratory system, constrictions in general and the various types of branch junction are important sites as regards blockage and disease initiation [1-32], while the networks established by branchings span the entire human body. Both (a), (b) affect cardiovascular performance in the human torso and cerebrovascular responses in the head, with the latter including Stroke, specifically arteriovenous malformations and the phenomenon of 'steal' in which blood flowing normally to one part of the brain is diverted away adversely to other parts [33-40]. The word 'constriction' in (a) is taken here to stand generally for constrictions, dilations, roughnesses, corners and related distortions of the vessel, while the 'branching' (b) may take many different forms. The fluid-flow properties including the induced wall shear stress and pressure play a vital role throughout and for (a), (b) are found to establish many of the basic principles of internal flows.

Item (a) in our context at least is felt to be more established [41-47]. It is also very enlightening, has exhibited (in [46,47] and subsequent references) encouraging comparisons and agreement between theory, computational simulations and experiments, is interesting with regard to both applied mathematics and fluid dynamics itself, and leads on naturally into (b). Item (b) is rather newer as regards rational theoretical treatment [48-50] and that leaves many substantial intriguing issues to be explored as we shall see. There being a great amount of practical interest has sparked off a great number of computational studies [51-58]. The current concern however is in seeking more mathematical progress, partly in order to complement computational as well as experimental work, partly for its own sake, partly to shed light on parameter dependence and partly in terms of possibly increasing total understanding of the wider subject area.

The viscous fluid contained within the typical vessel considered below is supposed to be incompressible with constant density, Newtonian and flowing in laminar fashion whether steadily or unsteadily in two or three spatial dimensions. The representative flow rate plays a crucial role in determining the flow properties and the theoretical solution, as does the vessel geometry specified under (a) or (b) above: see Fig. 1. That flow rate is characterised by the Reynolds number $R e$ which measures in essence the flow momentum effects relative to the viscous frictional forces. The value of $R e$ can be as large as several thousand in major arteries and it remains at least in the hundreds for many constrictions and branchings of present concern, for instance in the human torso and in the head. Mathematically the use of asymptotic approaches based on taking $R e$ as a large parameter has been found to work well in those ranges [59-63], often far more so than in aerodynamics for example. Other ranges are undoubtedly of much interest also including use of $R e$ as a small parameter for the study of capillary-scale influences [64-67]. Nevertheless our eventual focus in this work is on medium-to-high Reynolds numbers.

Possible practical limitations of the approach which can vary from case to case should also be mentioned. They centre mostly on turbulence, three-dimensionality, 


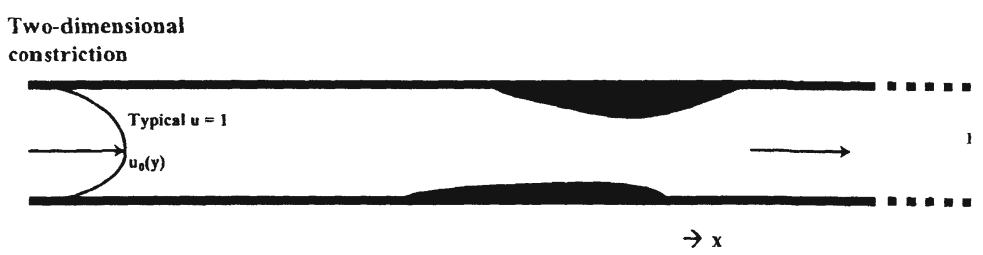

Three-dimensional

constriction /dilation
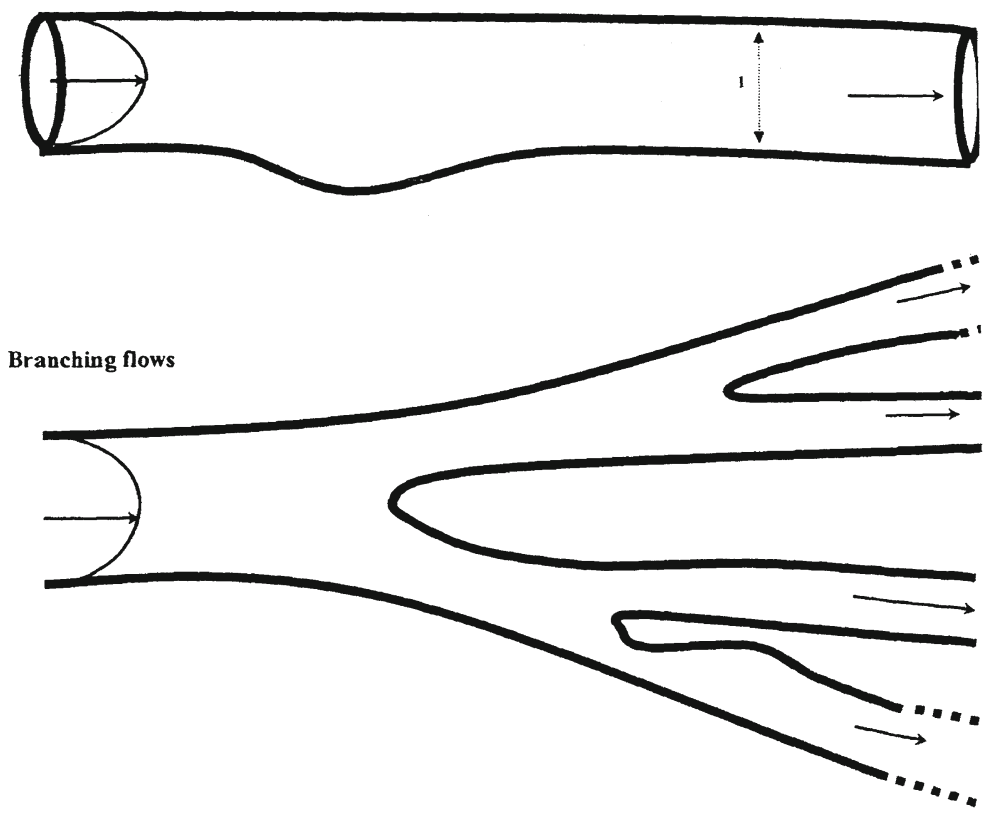

Fig. 1 Representative non-dimensional picture for internal fluid motion with a constriction or branching

non-rigid wall effects, non-Newtonian effects, wall roughness and the use of asymptotics. Flow transition to turbulence however occurs comparatively little in the human torso and even less so in the head; so the relevance of laminar flow theory to biomedical applications is potentially high, in contrast to the relevance in certain engineering applications. Along with this the importance of three-dimensional behaviour usually cannot be denied. In the present setting we seek solution structure and some increased understanding through studying the two-dimensional versions but use those to guide the beginnings of three-dimensional theory, specifically in Sect. 7 below. As regards the influences of non-rigid walls much theoretical work has been done or is under way, although we note again their limited influence in the head. Non-Newtonian effects are undoubtedly important at smaller length scales. Small roughness effects are almost always present in any motion past a solid surface and are the subject of continuing interest of course. Finally here, the use of asymptotic analysis is supported by, among other things, the desire to capture theoretically a wide range of scales and a considerable 
number of comparisons with experiments and direct numerical simulations as discussed earlier and as we shall see later in this article.

Section 2 below presents the major non-dimensionalised governing equations of motion that hold in the remainder of the article, along with the reasoning, the orders of magnitude and the scaling factors involved in the derivation of the equations within various parts of the vessels: see also the review in [68]. Section 3 then describes the theory and corresponding analysis for constrictions that are symmetric in two dimensions or axisymmetric in three dimensions; two later sections deal with cases of non-symmetry. A gradual increase in the strength of constriction is discussed mathematically leading to a progression from strong to moderate to severe constriction in which flow reversal and separation can come into play significantly. This is followed by Sect. 4 which extends the arguments to motion inside branching vessels over comparatively short length scales (relating also generically to several types of cardiovascular and cerebrovascular branching, including arteriovenous malformations) and Sect. 5 in which interactions between long and short length scales and/or between viscous and inviscid forces are of importance, again for branching flows, with attention being given to side-branching, large networks and viscous wall layers. Upstream influence which can arise from several different mechanisms is addressed in Sect. 6, covering especially the case of non-symmetry in two dimensions, while three-dimensional effects for non-axisymmetric constrictions or realistic branchings are the subject of Sect. 7. Final comments are made in Sect. 8, including suggested future project topics.

\section{Equations and scales}

Some initial words are appropriate here on the internal flow configurations to be discussed. The typical geometry which is that of a vessel or system of vessels and the typical incoming (incident) flow inside the vessel are explained by the diagram in Fig. 1, where laminar flow of the viscous incompressible fluid is assumed. The vessel of concern tends to be elongated axially to a greater or lesser extent and to be constricted, dilated, distorted or branched in various different ways as shown, while the incoming velocity profile in the fluid can be almost completely straight or significantly curved depending on flow properties ahead of the present configuration. The details of the configuration lead to many interesting distinct forms of response in the physical system which we hope to be able to capture through relevant mathematical systems or models. The work below will be general in three spatial dimensions first but after that mostly on two-dimensional behaviour, returning to the three-dimensional version near the end of the article. The flow velocity vector and components are defined as $\boldsymbol{u}^{*}=\left(u^{*}, v^{*}, w^{*}\right)$, with corresponding Cartesian coordinates $\left(x^{*}, y^{*}, z^{*}\right)$, the dynamic pressure $p^{*}$, the time $t^{*}$, as well as the uniform density $\rho^{*}$, viscosity $\mu^{*}$ and kinematic viscosity $\nu^{*}\left(=\mu^{*} / \rho^{*}\right)$ of the fluid. An asterisk refers to a dimensional quantity.

In the rest of this section we describe the main governing equations in Sect. 2.1, as these are to be used throughout the article, including especially the reduced systems, followed by Sect. 2.2 which is on the different scales present. 


\subsection{The governing equations}

In dimensional terms the governing equations for the fluid motion have the vector form

$$
\begin{aligned}
& \nabla^{*} \cdot \boldsymbol{u}^{*}=0, \\
& \rho^{*}\left[\partial_{t^{*}} \boldsymbol{u}^{*}+\left(\boldsymbol{u}^{*} \cdot \nabla^{*}\right) \boldsymbol{u}^{*}\right]=-\nabla^{*} p^{*}+\mu^{*} \nabla^{* 2} \boldsymbol{u}^{*} .
\end{aligned}
$$

These are respectively the continuity and the Navier-Stokes equations in Cartesian coordinates. The gradient operator $\nabla^{*}=\left(\partial_{x^{*}}, \partial_{y^{*}}, \partial_{z^{*}}\right)$. To rid ourselves of the effects of units which can be misleading when a rational reduced approach is required we prefer to work in terms of non-dimensional quantities, which provide more clarity. Thus we suppose a representative velocity to be $u_{r}^{*}$ say, a representative length to be $l_{r}^{*}$, and then write

$$
\left(x^{*}, y^{*}, z^{*}\right)=l_{r}^{*}(x, y, z), \boldsymbol{u}^{*}=u_{r}^{*} \boldsymbol{u}, p^{*}=\rho^{*} u_{r}^{* 2} p, t^{*}=\left(l_{r}^{*} / u_{r}^{*}\right) t,
$$

with $\boldsymbol{u}$ denoting $(u, v, w)$. Here $u_{r}^{*}$ would be the characteristic velocity amplitude of flow expected in the vessel and $l_{r}^{*}$ would be the characteristic distance measured laterally across the vessel, for example the radius of a vessel of uniform circular cross section. The time factor is taken as the convection-based time $l_{r}^{*} / u_{r}^{*}$ and the pressure factor as the convection-based value $\rho^{*} u_{r}^{* 2}$ for convenience. If an overall pressure gradient $K^{*}$ is given to us we might reasonably define $u_{r}^{*}$ as $\left(K^{*} l_{r}^{*} \rho^{*}\right)^{1 / 2}$ at this stage. The governing equations $(1,2)$ now become the nondimensional Navier-Stokes equations

$$
\begin{gathered}
\nabla \cdot \boldsymbol{u}=0, \\
\partial_{t} \boldsymbol{u}+(\boldsymbol{u} \cdot \nabla) \boldsymbol{u}=-\nabla p+R e^{-1} \nabla^{2} \boldsymbol{u},
\end{gathered}
$$

in turn, where $\nabla=\left(\partial_{x}, \partial_{y}, \partial_{z}\right)$ is the non-dimensional gradient operator. The present article will work in the context of the non-dimensional system $(4,5)$, which is subject to the boundary conditions

$$
\boldsymbol{u}=0 \text { on walls, }
$$

inflow (outflow) constraints far upstream (downstream),

as appropriate to internal flows. Here the requirement (6) is the no-slip condition holding on any fixed solid surface adjoining the fluid.

In (5), $R e=u_{r}^{*} l_{r}^{*} / v^{*}$ which is the Reynolds number of the flow measures the ratio of the representative inertial forces to the representative viscous forces. This is in line with the left-hand side of (5) representing inertia or convection along with acceleration, whereas viscous effects are on the right-hand side. In applications in biomedicine Re can range from rather large to extremely small values. This wide variation in values points to the possible benefit to be gained by use of reduced forms of the full system $(4,5)$. 
The main reduced forms, for the medium to large range of $R e$, are the Euler equations and the boundary-layer (or wall-layer) equations. The former stem from simply neglecting the viscous contributions in (5), yielding

$$
\partial_{t} \boldsymbol{u}+(\boldsymbol{u} \cdot \nabla) \boldsymbol{u}=-\nabla p,
$$

along with (4). If $\boldsymbol{u}, p, \nabla, t$ are generally of order unity then the inviscid Euler equations $(4,8)$ seem a sensible deduction from the full Navier-Stokes system when $R e$ is suitably large. More broadly perhaps a scaling of the form

$$
(x, y, z, t, u, v, w, p)=\left(\sigma_{1} x^{\prime}, \sigma_{2} y^{\prime}, \sigma_{3} z^{\prime}, \sigma_{4} t^{\prime}, \sigma_{5} u^{\prime}, \sigma_{6} v^{\prime}, \sigma_{7} w^{\prime}, \sigma_{8} p^{\prime}\right)
$$

applies where the factors $\sigma_{1}-\sigma_{8}$ are the scales that leave the primed (') variables all of order unity. We can immediately argue however that the typical $x$-wise scale $\sigma_{1}$ is itself unity if the spatial non-dimensionalisation has been chosen appropriately and likewise $\sigma_{2}, \sigma_{3}$ are of $O(1)$ in the general case, while $\sigma_{5}-\sigma_{7}$ are also of order unity for similar reasons associated with the characteristic velocity amplitude. Then substitution into (4), (5) leaves the continuity equation (4) standing as it is in terms of the primed variables and gives in the momentum equations (5) the orders

$$
\sigma_{4}^{-1}, 1 \text { on the left side and } \sigma_{8}, R e^{-1} \text { on the right, }
$$

in turn. Hence $\sigma_{4}, \sigma_{8}$ can both be expected to be of $O(1)$ also generally to provide a momentum balance at leading order when $R e$ is large. So in this comparatively simple case the scalings are

$$
\sigma_{n}=O(1) \text { for } n=1,8 \text {, }
$$

in line with (8). The influence here of the non-dimensionalisation should be noted of course, as well as the possibility of different distinct scales such that $\nabla$ may be of large amplitude in certain parts of the motion or the temporal scale $\sigma_{4}$ may be relatively small or large in certain prescribed-frequency problems. Also the neglect of viscous contributions, which contain the highest derivatives in the original system $(4,5)$, means in general that we can only apply part of the requirement $(6)$, the usual guess for this being the tangential-flow or zero-normal-velocity condition: if say the wall lies along $y=0$ then $v=0$ is required there, leaving $u, w$ as usually nonzero. That guess is tantamount to assuming there is no substantial departure of the Euler flow from the wall, i.e. the motion is separation-free. This also brings us on to the viscous boundary-layer equations which hold in relatively thin layers where (5) reduces to the form

$$
\begin{aligned}
\partial_{t}(u, w)+(\boldsymbol{u} \cdot \nabla)(u, w) & =-\left(\partial_{x}, \partial_{z}\right) p+R e^{-1} \partial_{y}^{2}(u, w), \\
0 & =-\partial_{y} p
\end{aligned}
$$


in three spatial dimensions or

$$
u_{t}+u u_{x}+v u_{y}=-p_{x}(x, t)+R e^{-1} u_{y y}
$$

in two dimensions, together with (4), for flow situations where the relative thinness is in the $y$-direction. The thinness accentuates the $y$-derivatives in the gradient operator, leading to the result in (13) that the leading-order pressure must be independent of the $y$ position and to the viscous effects in (12) being dominated by the $y$-derivatives as shown. More broadly again a scaling of the form (9) holds. Usually the typical $x$-scale $\sigma_{1}$ is unity as before, as is $\sigma_{3}$, but $\sigma_{2}$ is unknown, and the velocity scalings $\sigma_{5}, \sigma_{7}$ are also of order unity but $\sigma_{6}$ is unknown. Substitution into (4), (5) leaves the continuity equation (4) in suitable balance provided $\sigma_{2} \sim \sigma_{6}$ while the $x$ - and $z$-momentum equations within (5) yield the orders

$$
\sigma_{4}^{-1}, 1\left(\& \sigma_{6} \sigma_{2}^{-1}\right) \text { on the left side and } \sigma_{8}, R e^{-1} \sigma_{2}^{-2} \text { on the right. }
$$

Thus again $\sigma_{4}, \sigma_{8}$ might be expected to be of $O(1)$ generally whereas now the $y$-scaling $\sigma_{2} \sim \sigma_{6} \sim R e^{-1 / 2}$ is implied. So here the scalings essentially have

$$
\sigma_{n}=1 \text { for } n=1,3,4,5,7,8 \text {, with } \sigma_{2}=\sigma_{6}=R e^{-1 / 2} \text {. }
$$

Strictly we then insert the transformation (9) together with (16) in (4), (5), to obtain

$$
\begin{gathered}
\nabla^{\prime} \cdot \boldsymbol{u}^{\prime}=0, \\
\partial_{t^{\prime}}\left(u^{\prime}, w^{\prime}\right)+\left(\boldsymbol{u}^{\prime} \cdot \nabla^{\prime}\right)\left(u^{\prime}, w^{\prime}\right)=-\left(\partial_{x^{\prime}}, \partial_{z^{\prime}}\right) p^{\prime}+\partial_{y^{\prime}}^{2}\left(u^{\prime}, w^{\prime}\right)+o(1), \\
O\left(R e^{-1}\right)=-\partial_{y^{\prime}} p^{\prime},
\end{gathered}
$$

and take the limit of (17)-(19) as $R e$ tends to infinity with all the primed variables remaining of $O(1)$. This reproduces (12), (13) in effect. The validity of the boundary layer system $(12,13)$ relies mostly on a balance $|u|^{2} /|x| \sim R e^{-1}|u| /|y|^{2}$ being struck between inertial and viscous contributions in the sense of orders of magnitude, provided that the acceleration and pressure contributions respond passively to the others. If $u$ is typically of $O(1)$ for instance then we obtain the classical scaling

$$
|y| \sim R e^{-1 / 2}|x|^{1 / 2}
$$

for the 'thickness' [69-76] of the relatively thin boundary layer. A subset of $(12,13)$ gives another reduced form, specifically (4), (13) again but with

$$
0=-\left(\partial_{x}, \partial_{z}\right) p+R e^{-1} \partial_{y}^{2}(u, w)
$$

which is the lubrication approximation. This has negligible acceleration and inertial forces from comparison of (12), (21) and is valid subject to the main assumption that $|u|^{2} /|x| \ll R e^{-1}|u| /|y|^{2}$, i.e. $R e \ll|x||u|^{-1}|y|^{-2}$, which can be interpreted as requiring a reduced Reynolds number to be small. The form (21) can also be deduced 
from the Navier-Stokes system $(4,5)$ by means of a scaling as in (9). The latter leads to the orders displayed in (15) but now the scaling $\sigma_{2}=\sigma_{6} \ll R e^{-1 / 2}$ is implied, followed by a strict transformation and limit process which yield (21) effectively. The relevant boundary conditions for $(12,13)$ or $(21)$ depend on the specific physical setting as we shall see later.

By contrast the main reduced form appropriate for small $R e$ values is based on the Stokes equations

$$
0=-\nabla p+R e^{-1} \nabla^{2} u
$$

and (4), with acceleration and inertial effects being suppressed in comparison with (5). The pressure $p$ here would usually be anticipated as being of order $R e^{-1}|u| /|x|$. The background scaling (9) leads to the orders $\sigma_{5} \sigma_{4}^{-1}, \sigma_{5}^{2} \sigma_{1}^{-1}$ on the left side in (5) and $\sigma_{8} \sigma_{1}^{-1}, R e^{-1} \sigma_{5} \sigma_{1}^{-2}$ on the right, for $\sigma_{1} \sim \sigma_{2} \sim \sigma_{3}$. So provided $\sigma_{4}$ is not too small and $\sigma_{5}$ not too large the system (22) is inferred; this can be confirmed by a strict transformation and limiting process as above which yield (22) in terms of the primed variables. The lubrication approximation $(4,13,21)$ can still apply here for thin vessels.

Our interest below centres mostly on the range where the values of the Reynolds number $R e$ are medium to large. Partial derivatives with respect to $y$ say will be written as $\partial_{y}$ or $\partial / \partial y$ or with a subscript $y$, and similarly for other coordinates, depending on the specific context, and a prime will denote an ordinary derivative. Also in two-dimensional flows (4) leads to the existence of a stream function $\psi$ such that $u=\partial \psi / \partial y, v=-\partial \psi / \partial x$ subject to an additive constant.

\subsection{Long and short scales}

The derivations and justifications of the key governing equations above hold true to leading order. The full approach is expressed more systematically in terms of asymptotic expansions for specific contexts in Sect. 3 onwards. It soon becomes clear (see Fig. 2) for many two-dimensional steady configurations that at least three major length scales can apply readily to the internal flows of concern at medium to large $R e$ values. This holds quite generally for distorted channel flows say with constrictions, dilations, roughnesses or corners although we also keep the application of branching flows firmly in mind.

First, one such scale arises from consideration of the steady flow in a long vessel which is a straight or nearly straight channel with axis in the $x$ direction. See Fig. 1 which acts to define the $x, y$ coordinates within the channel as axial and lateral respectively. Seeking in effect a unidirectional flow solution in the sense that the $y$-velocity component $v$ is zero (or negligible) with $u, p$ nonzero leads to $u=u(y)$ being independent of $x$ from the continuity equation (4) whereas $\partial p / \partial y=0$ from the lateral $y$-momentum equation within (5). Further, the $x$-momentum equation within (5) then has an identically zero left-hand side and requires $\partial p / \partial x$ to be independent of $x$, i.e. the axial pressure gradient is uniform. Thus the governing equations here take the form 

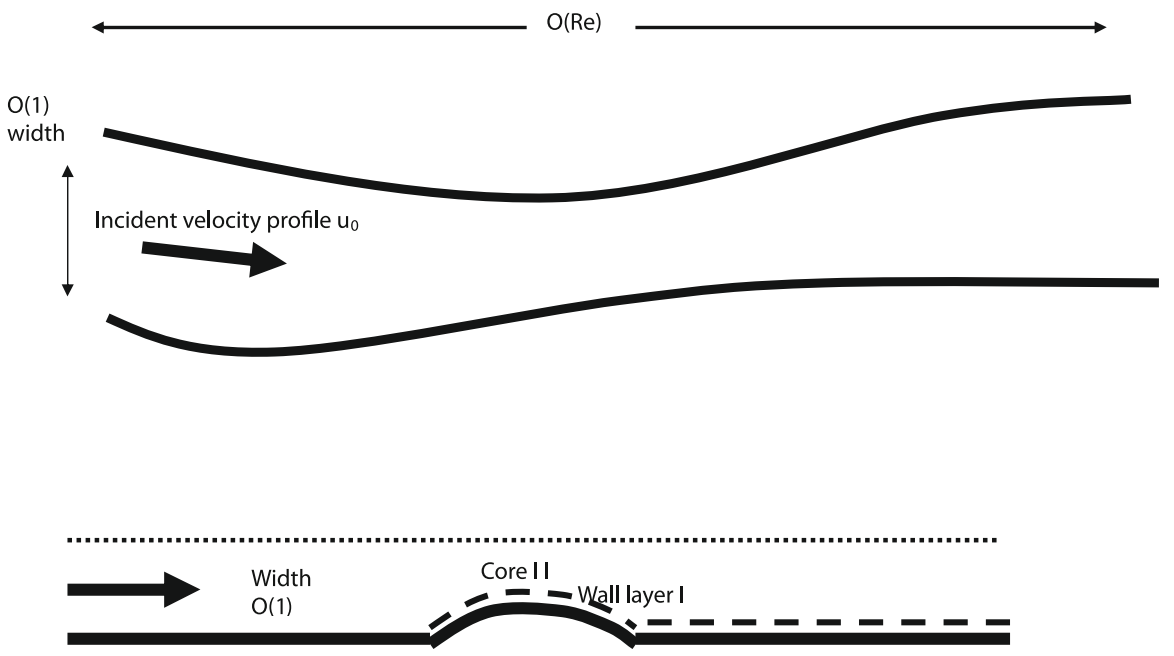

Constriction

length $O(1)$

(a)

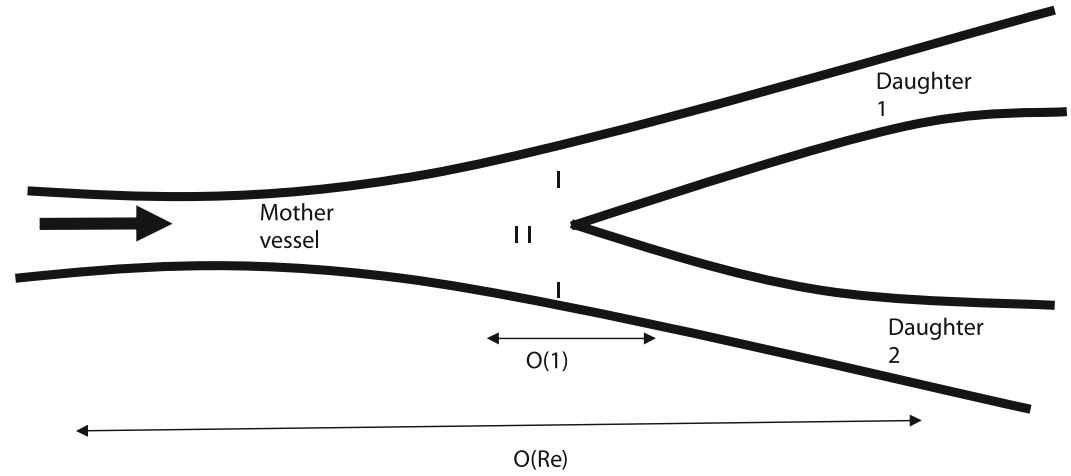

(b)

Fig. 2 Length scales and typical flow structure for (a) constriction, (b) branching, including viscous wall layer I and inviscid core II

$$
\begin{aligned}
& 0=0, \\
& 0=-\partial p / \partial x(x)+R e^{-1} d^{2} u / d y^{2}, \\
& 0=-\partial p / \partial y+0
\end{aligned}
$$

compared with $(4,5)$. Given (25), the partial derivative in (24) can be replaced by a full derivative. The exact solution for $u$ therefore gives the parabolic velocity profile

$$
u(y)=\frac{1}{2}\left(y-y^{2}\right)(-\operatorname{Re} d p / d x)
$$


of the well-established plane Poiseuille flow in a straight channel with $y=0,1$ at the walls.

The result (26) is usually interpreted as predicting the fluid motion in a straight channel when subject to a prescribed axial pressure gradient. What matters perhaps most here in the broader context is that the pressure gradient must scale with $R e^{-1}$ in order for $u$ to be typically of $O(1)$, as it should be by definition, and so if the representative pressures imposed at the far upstream and far downstream ends of the channel are say $p=p_{0}, p=p_{1}$ in turn with the pressure difference being of order unity in general then the axial length scale $x$ must be $O(R e)$. This length scale of order $R e$ is a main long scale.

The velocity $u$ in (26) along with zero $v$ and constant $d p / d x$ gives an exact solution not only of the Navier-Stokes equations $(4,5)$ but also of the boundary layer equations $(12,13)$ by virtue of the balances remaining in (23-25) as well as the subset in (21). The boundary layer system reinforces the importance of the $O(R e)$ length scale, while the lubrication system tends to correspond to longer length scales. The same $O(R e)$ length scale is supported by an argument based on orders of magnitude as discussed earlier towards the end of Sect. 2.1. It gives us viscous-inviscid balances acting laterally across the entire vessel. The advantage of the order-of-magnitude argument is that it applies to a wider range of vessel shapes, allowing for significant distortion for instance.

Second, another major scale is apparent from the geometry of most branching junctions themselves or more generally from the vessel width. This is a local-flow scale as indicated in Fig. 2. For a constriction or a one-to-two branching for example we can expect the $O(1)$ axial scale to play a strong role as it yields the Euler equation (8) at leading order which are inviscid and elliptic and thus it provides a mechanism for upstream influence in the neighbourhood of the constriction or junction (see also Sects. 4, 6 later) and, more importantly in terms of branching, 'jumps' acting over the $O$ (1) length scale. Such constrictions are considered later in this article in Sect. 3. As regards branching here we work with $y$ defined laterally across each vessel or subvessel and with the 'mother' vessel being upstream followed successively by daughter sub-vessels, grand-daughters, etc downstream. Suppose we have so-called plug flow in which the velocity profile $u_{0}(y)$ upstream in the mother tube is uniform and nonzero in $y$; such a profile is often acceptable for an inviscid flow account as hinted in Sect. 2.1. Then the effective vorticity $\boldsymbol{\nabla} \times \boldsymbol{u}$ is zero throughout the local flow if that flow remains separation-free, as explained in the next section, and so we can anticipate plug flows with constant $u=u_{1}, u=u_{2}$ emerging in the two daughters downstream. The values of the velocities $u_{0}, u_{1}, u_{2}$ are unknown in advance since the motion here is driven by prescribed pressures $p=\pi_{0}$ in the mother and $p=\pi_{1}, p=\pi_{2}$ in the two daughters. The conditions to determine the velocities stem from integrals acting on the governing equation (8) which yield conservation of the Bernouilli quantity $p+\frac{1}{2} u^{2}$ along streamlines since

$$
\left\{\partial_{t}+(\boldsymbol{u} \cdot \nabla)\right\}\left(p+\frac{1}{2} u^{2}\right)=0
$$

and hence the overall requirements of conservation of mass and momentum in the form 


$$
\begin{aligned}
& u_{1} h_{1}+u_{2} h_{2}=u_{0} h_{0}, \\
& \pi_{1}+\frac{1}{2} u_{1}^{2}=\pi_{2}+\frac{1}{2} u_{2}^{2}=\pi_{0}+\frac{1}{2} u_{0}^{2} .
\end{aligned}
$$

Here $h_{0}, h_{1}, h_{2}$ are the respective vessel widths. The three algebraic equations (28-29) control the three unknowns $u_{0}, u_{1}, u_{2}$ and are readily solved in principle subject to the implicit assumption that the velocity components are positive. If there are more than two daughter branches at the junction, on the other hand, or if there are further generations of branchings (daughters, grand-daughters and so on in a tree-like structure), or if other length scales come into play, then solving is more difficult and involved. Nonlinear coupled recurrence relations and coupled differential equations for example can arise. We defer these matters to later on, mentioning for now the main point that the above confirms the importance of the local length scale of $O(1)$.

Third, however, there are numerous length scales which are potentially significant in-between the two above. In such cases we have a boundary or wall layer near any wall and an inviscid core in the rest of the flow field. If the core gives an $O(1)$ slip velocity then the wall-layer thickness scales as $R e^{-1 / 2}|x|^{1 / 2}$ as reasoned in Sect. 2.1 whereas if the core yields say u of $O(y)$ as in (26) especially then the wall layer has $y$-scale

$$
|y| \sim R e^{-1 / 3}|x|^{1 / 3}
$$

by a similar reasoning. Often the wall layer and the core affect each other about equally, giving viscous-inviscid interaction.

Fourth, length scales can also lie also outside the range described above of course. For example the lubrication mechanism (21) applies for lengths $|x|$ greater than $O(R e)$, and indeed generally these outside length scales yield subsets of the main problems above.

From now on the article concentrates mostly on major examples and their features.

\section{Constrictions}

The mathematical study of constriction effects here is founded on [77], begins with symmetric cases and includes flow separation and eddies. The study extends to nonsymmetric and three-dimensional constrictions in later sections and it also takes us suitably on to recent investigations of branching effects in the following section.

We concentrate then on the steady motion of an incompressible fluid through a symmetrically constricted channel or axisymmetric-pipe when $R e$ is asymptotically large. Here for channel flows along with the Navier-Stokes equations $(4,5)$ we have

$$
u \rightarrow y-y^{2}, \quad v \rightarrow 0, \quad p \sim-\frac{2 x}{R e} \text { as } x \rightarrow-\infty
$$

if for definiteness we assume Poiseuille flow (26) far upstream between the undisturbed channel walls $y=0,1$ : see also (7). Generalisations of these conditions are possible. 


\subsection{Strong constriction}

One of the procedures helpful here for a particular form of disturbance (constriction, corner, hump, etc.) is to consider the flow response for increasing sizes of the disturbance and then to single out that first crucial size, the strong disturbance, which produces a non-linear response with unknown pressure, so that among other things any separation encountered may be regular. Separation or flow reversal can usually occur only in a fully nonlinear process. By way of aside, in external flows the most crucial size is the triple-deck one as order of magnitude arguments confirm [78]. In symmetrically constricted internal flows the crucial height ("strong" constriction) is as in (30).

Take first $|x|=O(1)$ and the symmetric channel case, again for definiteness: nonsymmetric channel flows are discussed in Sect. 6, while other sizes of $|x|$ are mentioned below, and axisymmetric pipe flows have the same structure as here. Then with the strong constriction given by $y=h R e^{-1 / 3} F(x)$ at the lower wall and $0<h<\infty$, the flow near it, in the viscous wall layer I of Fig. 2, is described by the asymptotic expansions

$$
(u, \psi, p)=\left(R e^{-1 / 3} U, R e^{-2 / 3} \Psi, \operatorname{Re}^{-2 / 3} P(x)\right)+\cdots, y=R e^{-1 / 3} Y, \quad x=O(1),
$$

in view of the oncoming form (31) and the required inertia-pressure-viscous force balance near the constriction. Substituting into the Navier-Stokes equations $(4,5)$ and keeping track of the scaling factors then gives

$$
R e^{-1 / 3} U_{x}+\cdots-R e^{-1 / 3} \Psi_{x Y}+\cdots=0,
$$

in (4) since $v=-\psi_{x}$, while in (5) the $x$ component has

$$
R e^{-2 / 3} U U_{x}+\cdots-\operatorname{Re}^{-2 / 3} \Psi_{x} U_{Y}+\cdots=-\operatorname{Re}^{-2 / 3} P_{x}+\operatorname{Re}^{-1} \operatorname{Re}^{1 / 3} U_{Y Y}+\cdots,
$$

and the $y$ component yields

$$
O\left(R e^{-1}\right)=-\operatorname{Re}^{-1 / 3} P_{Y}+\cdots,
$$

since the flow is steady. It follows at leading order that $\mathrm{P}$ is independent of $\mathrm{Y}$ as anticipated in (32) and we are led to the boundary-layer equations $(4,14)$ for $U, \Psi, P$, with $U=\Psi_{Y}$ and $R e$ replaced by unity. Here the pressure $P(x)$ is unknown, while the boundary conditions are

$$
\begin{aligned}
& (U, \Psi, P) \rightarrow\left(Y, \frac{1}{2} Y^{2}, 0\right) \text { as } x \rightarrow-\infty[\text { from (31)], } \\
& U=\Psi=0 \text { at } Y=h F(x)[\text { from }(6)], \\
& U \sim Y+0 \text { as } Y \rightarrow \infty\left[\Rightarrow \Psi \sim \frac{1}{2} Y^{2}+P(x)\right] .
\end{aligned}
$$



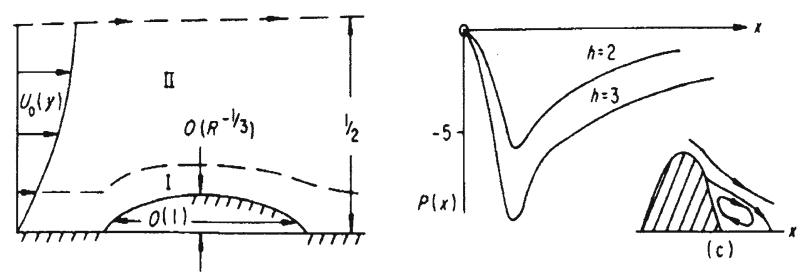

(i)

(a)

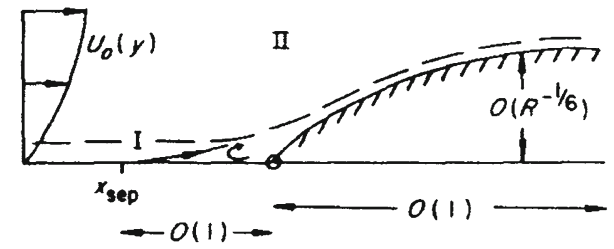

(b)

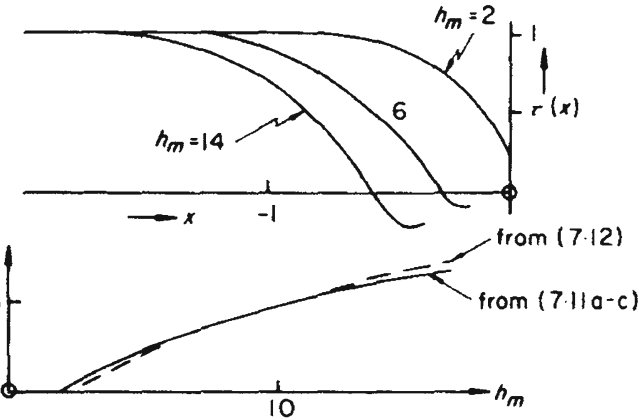

(ii)

Fig. 3 Symmetric tube-flow. (i) With a strong constriction, showing structure, pressure solutions and typical streamlines (in (c)), where $R, U_{0}$ denote $R e, u_{0}$ respectively. (ii) With a moderate constriction, showing structure, wall shear stress $r$ upstream and the upstream separation position; here (7.11a-c), (7.12) correspond to our (51-53), (54) respectively

The lack of displacement (cf. the presence of $A(x)$ in triple-deck theory $[68,77,78]$ and below) in $U$ in (38) is necessary for the satisfaction of the symmetry condition at $y=\frac{1}{2}$ in the core $(0<y<1)$ of the flow. The non-linear problem with (36-38) is immediately a closed one, therefore, and solutions for $h \ll 1$ (weak constriction) and for $h=O(1)$ (strong constriction) are obtained as in Fig. 3, fixing the pressure distribution $P(x)$; see also figure 23.4 of [82] and observe there is clearly no upstream influence yet. The same analysis applies to dilations $F(x) \leq 0$ but only constrictions $F(x) \geq 0$ are discussed henceforth. The pressure gradient is then favourable initially as the fluid is pressed against the front of the constriction, but on the leeward side the pressure rises, the skin friction falls rapidly and for sufficiently large $h$ regular separation occurs. The pressure then remains negative and its variation is quite small in the ensuing reversed flow, until reattachment takes place with the pressure $P$ thereafter rising to its ultimate downstream value of zero. Next, in the inviscid core II it is deduced from the behaviour in the viscous layer that 


$$
(u, \psi, p)=\left(y-y^{2}, \frac{1}{2} y^{2}-\frac{1}{3} y^{3}, 0\right)+\operatorname{Re}^{-2 / 3}\left(u_{1}, \psi_{1}, p_{1}\right)+\cdots,
$$

for $x, y$ of order one. A substitution process as in (33-35) above then applies. So $\psi_{1}$ is found to satisfy the linear elliptic equation

$$
u_{0}\left(\frac{\partial^{2} \psi_{1}}{\partial x^{2}}+\frac{\partial^{2} \psi_{1}}{\partial y^{2}}\right)=u_{0}^{\prime \prime} \psi_{1}
$$

from $(4,5)$, with $u_{0}(y) \equiv y-y^{2}$ and boundary conditions

$$
\begin{aligned}
& \psi_{1}=0 \text { at } y=\frac{1}{2} \text { (for symmetry), } \\
& \left.\psi_{1} \rightarrow 0 \text { as } x \rightarrow-\infty \text { (from }(31)\right) \\
& \text { no exponential growth as } x \rightarrow \infty \\
& \psi_{1} \rightarrow P(x) \text { as } y=\rightarrow 0+(\text { to match with } \mathrm{I})
\end{aligned}
$$

Solutions for $\psi_{1}$ are given by [42].

We notice (a) the ordering of the above asymptotic scheme: solve the viscous nonlinear problem in region I first, for $P(x)$; then solve in the inviscid region II for $\psi_{1}$, and so on. The ordering is the complete opposite of that originally proposed in classical treatments for external flows. Here the viscous wall layer fixes the wall pressure distribution and the wall pressure then drives the small inviscid perturbation in the core flow by means of its displacement effect in (44). (b) The regularity of the solution in region I at any separation. (c) The small size of the coreflow disturbance in (39). (d) The absence of any non-linear upstream response. For, if the constriction starts at $x=0$ as we shall assume henceforth, then

$$
U=Y, \quad \Psi=\frac{1}{2} Y^{2}, \quad P(x)=0 \text { for } x<0
$$

The upstream response is only of a linear kind and is due to the feedback from the ellipticity in the inviscid coreflow II; it is worth considering nevertheless. The solution of (40-44) gives the typical inviscid slip property

$$
u_{1} \rightarrow A_{1}(x) \text { as } y \rightarrow 0+\text {, in } x<0 \text {, }
$$

in view of (44) with (45). Here the slip velocity or negative displacement $A_{1}(x)$ is a given function determined from (40-44) (see e.g. (53) below). Hence, upstream $(x<0)$ in the viscous layer in I again,

$$
(u, \psi, p)=\left(\operatorname{Re}^{-1 / 3} Y, \frac{1}{2} R e^{-2 / 3} Y^{2}, 0\right)+\left(R e^{-2 / 3} U_{1}, \operatorname{Re}^{-1} \Psi_{1}, \operatorname{Re}^{-1} P_{1}(x)\right)+\cdots
$$


and from substitution as in (33-35) again $U_{1}, \Psi_{1}, P_{1}$ satisfy linear viscous equations of motion with the outer boundary condition

$$
U_{1} \sim-Y^{2}+A_{1}(x) \text { as } Y \rightarrow \infty
$$

So the upstream wall layer response is linear and in particular upstream separation is not possible yet.

However, in the spirit of this investigation, suppose we let $h$ become large now and look for the trends of the flow solutions in the regions I, II above. It can be shown mainly from (38) in fact that $P(x) \sim-\frac{1}{2} h^{2}(F(x))^{2}$ when $h$ is large, at least if $F^{\prime}(x) \geq 0$. The limit $h \rightarrow \infty$ here forms the basis for the study in [79]. So then the core perturbation $\psi_{1}$ becomes large and $O\left(h^{2}\right)$, from the wall condition (44), implying for the upstream slip velocity $A_{1}(x)=O\left(h^{2}\right)$ also. Therefore in the upstream viscous response (47) the induced second-order perturbation $R e^{-2 / 3} U_{1}$ becomes $O\left(h^{2} R e^{-2 / 3}\right)$, from (48), and so is expected to become comparable with the leading term $\operatorname{Re}^{-1 / 3} Y$ of the velocity $u$ when $h$ grows to order $R e^{1 / 6}$, formally.

\subsection{Moderate constriction}

We consider next, therefore, a symmetric "moderate" constriction of length $O(1)$ still but of height $O\left(R e^{-1 / 6}\right)$, given by $y=h_{M} R e^{-1 / 6} F(x)$, where now $0<h_{m}<\infty$. The core flow II of Fig. 2 then suffers a larger perturbation than before, $R e^{-1 / 3}$ replacing $R e^{-2 / 3}$ in (39), but this leads again to (40-43) for $\psi_{1}$, although the wall constraint

$$
\psi_{1} \rightarrow \frac{1}{2} h_{M}^{2}(F(x))^{2} \text { as } y \rightarrow 0+
$$

holds instead of (44). The constraint (49) follows either from the suggestion above that $P \sim-\frac{1}{2} h^{2} F^{2}$, or it can be regarded simply as a Taylor series expansion of the typical inviscid condition $\psi \rightarrow 0$ as $y \rightarrow h_{M} R e^{-1 / 6} F(x)+$. The solution of (40-43) with (49) gives the upstream form (46) again, and so in the viscous wall layer I upstream

$$
(u, \psi, p)=\left(\operatorname{Re}^{-1 / 3} U, \operatorname{Re}^{-2 / 3} \Psi, \operatorname{Re}^{-2 / 3} P(x)\right)+\cdots(\text { for } x<0 \text { only }) .
$$

Here, from $(4,5), U, \Psi, P$ satisfy the non-linear problem of (36-38) except that

$$
\begin{aligned}
& U=\Psi=0 \text { at } Y=0, \\
& U \sim Y+A_{1}(x) \text { as } Y \rightarrow \infty,
\end{aligned}
$$

since $F(x)=0$ for $x<0$ and from (46). Here, we repeat, the negative displacement $A_{1}(x)$ is a known function of $x$.

Other details of the flow structure for this size of disturbance can be worked through but the upstream effect is the most vital for the following reasons. The displacement function $-A_{1}(x)$ is given by the infinite series $[79,80]$ 


$$
-A_{1}(x)=\sum_{n=1}^{\infty} \kappa_{n} \exp \left(\gamma_{n} x\right)
$$

where the constants $\kappa_{n}$ depend on the specific constriction shape $h_{M} F(x)$ and the constants $\gamma_{n}$ are the ordered eigenvalues of (40-42) with (49):

$$
0<\gamma_{1}<\gamma_{2}<\gamma_{3}<\ldots, \quad \text { e.g. } \gamma_{1}=5.175 \text {. }
$$

Analytical and numerical solutions of (36) with (51-53) as in [79] show that first, as $h_{M} \rightarrow 0$, a match with the structure of (32)-(48) for strong constriction is achievable; second, for $h_{M}$ of $O(1)$ and sufficiently large, upstream regular separation can occur, at $x=x_{\text {sep }}$ say; and, third, when $h_{M} \rightarrow \infty$, the core feedback through (52-53) becomes so severe that this separation is pushed indefinitely far upstream, $x_{\text {sep }} \rightarrow-\infty$. In fact, consideration of the exponential series in (53) gives us the asymptotic form

$$
x_{\text {sep }} \sim-\frac{2}{\gamma_{1}} \ln \left(h_{M}\right)+O(1) \text { as } h_{M} \rightarrow \infty,
$$

since $\kappa_{1} \propto h_{M}^{2}$. Thus the separation far upstream is a form of free interaction, as the specific value of $\kappa_{1}$ affects only the $O(1)$ contribution or origin shift in (54). Far upstream for $h_{M}$ large $-A_{1}(x) \sim \kappa_{1} \exp \left(\gamma_{1} x\right)$ and from the regular separation process then a pressure rise of order $R e^{-1 / 3}(P$ of $O(1))$ is produced overall, with a breakaway separation and structure like that of external flow [77,81,82] emerging downstream. So once again the upstream effect starts to change the whole flow structure.

\subsection{Severe constriction}

The form (54) provides the key to the flow structure for "severe" constriction, of length and height both $O(1)$. Formally, for a severe constriction, $h_{M}$ must grow to $O\left(R e^{1 / 6}\right)$ and so (54) implies separation at a large distance

$$
-x=-x_{\text {sep }}=\frac{1}{3 \gamma_{1}} \ln R e+O(1)
$$

upstream.

The suggestion (55) has been verified by a structural analysis [83] of severely constricted symmetric flows. Breakaway separation occurs both upstream, as the free interaction near (55), and on the constriction, via the incompressible triple-deck breakaway $[77,81,82]$. The Euler equations $(4,8)$ control the core of the motion, giving conservation of vorticity along streamlines there, but free streamline conditions of constant pressure hold along the unknown separated streamlines $\psi=0$ present both upstream and downstream. Between the free streamlines and the wall the recirculatory motions remain essentially inviscid and slow. The downstream reattachment occurs far beyond the constriction, when $x$ is $O(R e)$ and the boundary layer equations hold 
because $y, u, p$ are all $O(1)$ : see Sect. 2.2. Hence viscous forces can prevent the emergence of a significant recirculating back flow on the body scale $x=O(1)$. Again, on the constriction the reattachment of the upstream separated shear layer originating from (55) has only a minor influence on the dominant flow solution since its thickness rises only slightly from $O\left(R e^{-1 / 3}\right)$ when (55) holds to $O\left(R e^{-1 / 3}(\ln R e)^{1 / 3}\right)$ when $x$ is $O(1)$. The shear layer velocities are comparable with its thickness and so even a backward jet emanating from reattachment would force only a small pressure variation. The separated flow strategy in internal flow therefore appears complete. Between the first reattachment and the second, triple-deck, separation downstream on the constriction the boundary layer has the classical thickness $O\left(R e^{-1 / 2}\right)$ and is subjected to a favourable pressure gradient until the sudden breakaway at the triple-deck. Beyond this the $O\left(R e^{-1 / 2}\right)$ separated shear layer surrounds the free streamline $\psi_{0}=0$, at a constant $O(1)$ negative pressure, and ultimately on the body scale as $x \rightarrow \infty$ it becomes parallel to, while remaining detached from, the undisturbed channel wall. Solutions for moderately severe, very severe and slowly varying severe constrictions, and comparisons with experiments and Navier-Stokes calculations, are given by [83] for axisymmetric pipe flow, while $[84,46]$ compare Navier-Stokes solutions with the corresponding theory for symmetric channel flow; see also figure 23.5 of [82]. The comparisons are quite favourable.

Other relevant points are the following. First, if the constriction decays sufficiently slowly upstream rather than starting abruptly then the upstream separation can be suppressed. Second, a related study of bifurcating channel flow with a single channel flow dividing into two is given by [85]. We refer forward here to the next section. Third, there is the question of the flow features produced by a constriction whose dimensions do not fit exactly with the crucial scales assumed above. Broadly, the procedure is to suppose that the constriction length $l$ is given, with $O(1) \leq l \ll R e$, say. Then if the constriction height $H$ is of order $h_{c} \equiv R e^{-1 / 3} l^{1 / 3}$, the earlier work applies. So if the height is less than $O\left(h_{c}\right)$, a linearized version of the earlier work applies instead, involving the small parameter $H / h_{c}$. Conversely, if $H$ is greater than $O\left(h_{c}\right)$, we must appeal to the above reasoning and expect a substantial upstream response to be present. If $l$ is $O(1)$, the work of above then applies directly or in linearized form. If the constriction is long, $1 \ll l \ll R e$, then the flow structures of this section still control matters but, since the main structural length scale is of $O(1)$, only the initial constriction shape on the $O(1)$ length scale influences the upstream response. Therefore, the upstream response depends on whether this long constriction starts smoothly, or as a corner, or more abruptly. In any case, further downstream on the $O(l)$ length scale the flow properties become slowly varying. Similar considerations apply to short-scale constrictions as in Sect. 2 and to the non-symmetric motions considered in Sect. 6.

The above study for constrictions brings us appropriately on to consideration of branching next.

\section{Branching over short scales}

Concerning two-dimensional branchings, in this section we consider the $O$ (1)-length problem, i.e. 


$$
|x| \sim 1
$$

governed mainly by the inviscid system $(4,8)$ and assuming there is no separation (a matter discussed in Sect. 3 and below) as such an approach is found to be potentially useful. This could be with or without vorticity $\varsigma$ (defined as $-u_{y}+v_{x}$ )which is conserved along streamlines; if $\varsigma$ is zero then $(4,8)$ lead to Laplace's equation $\nabla^{2} \psi=0$ for the stream function $\psi$ and hence to a velocity potential $\varphi$ and to complex variable theory, which is applied later. This also supports the form (27-29) given earlier.

\subsection{Exact solutions}

An exact solution can be obtained analytically for the whole branching flow field in some cases, to check out recurrence results such as (28-29). A central example is the simple 1-to-2 bifurcation with mother walls a unit distance apart far upstream, daughters of width $h_{1}, h_{2}$ diverging at an angle $\alpha$ far downstream and straight sections in between along with respective velocities $1, u_{1}, u_{2}$. The two-dimensional problem is essentially as in [86] while the analogous three-dimensional problem is tackled later in Sects. 7.2, 7.3. The far-field velocities are related to the imposed pressures and to each other by means of (28-29) in two dimensions. The flow situation then can be resolved using a conformal mapping based on the Schwarz-Christoffel technique giving

$$
d z / d \chi=-\pi^{-1}\left\{(\chi+1)^{-1}-u_{1} h_{1}(\chi+b)^{-1}+\left(u_{1} h_{1}-1\right)(\chi-c)^{-1}\right\} e^{i \alpha} \chi^{-\alpha / \pi},
$$

where complex $z=x+i y$ and real $b=u_{1}^{\pi / \alpha}, c=u_{2}^{\pi / \alpha}$ are constants. The form (57) acts to determine the complex $\chi$ coordinate for a given $z$. Then the complex potential $w=\varphi+i \psi$ follows from

$$
w=\pi^{-1}\left[\ln \{(\chi+1) /(\chi-c)\}-u_{1} h_{1} \ln \{(\chi+b) /(\chi-c)\}\right] .
$$

The velocity profiles from the solution can be evaluated and are similar to the profiles presented in Sect. 7.3 below, clearly confirming the existence of upstream influence on the length scale of (56) with the spatial decay rate being $1 / \pi$.

Some other exact cases can be found by a similar technique. Most other cases however are largely computational depending on the particular geometry involved.

\subsection{Different features}

The vorticity $\varsigma$ is fixed by the input velocity profiles of the far field if there is no eddying present; this is supposing that as in Sect. 4.1 viscous effects are ignored. With nonzero vorticity $\nabla^{2} \psi$ is no longer zero or even simple in general and so in such circumstances computational simulation is usually necessary. With constant $\varsigma$ however some progress can be made based on solving $\nabla^{2} \psi=$ constant. 
More study can also be undertaken on the nonlinear recurrence relations such as $(28,29)$, for example for more daughters. In the limit of many daughter vessels being present the balance $(28,29)$ is replaced by

$$
\int_{(0,1)}\left\{2\left(\pi_{0}-p(y)\right)+u_{0}^{2}\right\}^{1 / 2} H(y) d y=u_{0} h_{0},
$$

where $p(y)$ denotes the given pressure distribution far downstream across the array of daughter vessels, $d y$ comes from a uniform stepping across the array normalised here so that $0<y<1, H(y)$ plays a density role in governing the lateral variation in gap width downstream, while $u_{0}$ is again the unknown uniform velocity in the mother far upstream; compare Sect. 4.4. For example if $p(y)=-\frac{1}{2} y p_{1}$ for a given constant $p_{1}$ and $H$ is unity throughout then provided $T, T+p_{1}$ are positive the form

$$
\pi_{0}=\frac{1}{2} T-2\left[\left(T+p_{1}\right)^{3 / 2}-T^{3 / 2}\right]^{2}\left(3 h_{0} p_{1}\right)^{-2} \text { with } T=2 \pi_{0}+u_{0}^{2}
$$

relates $u_{0}$ implicitly to the pressure effects $\pi_{0}$ and $p_{1}$. Solutions of the assumed form exist over a wide range of conditions.

Related progress on various other different features as presented in Fig. 4(i,ii) is addressed below.

\subsection{Linear/nonlinear effects}

Multiple branchings with small pressure changes are considered here. The geometry includes many dividers; each divider has a comparatively thin (Blasius [69]) boundary layer of thickness $O\left(R e^{-1 / 2}\right)$ from (22) as opposed to the slightly thicker $R e^{-1 / 3}$ layer at the outer wall implied by (30).

This work on the influence of small pressure differentials leads into the studies in the following sections, as well as into a comparison with direct simulations in this section. It assumes a nonuniform incident velocity profile $u=u_{0}(y)$ with zero slip at the walls in the single mother tube upstream and a branching (starting at $x=0$ ) into $N$ daughter tubes downstream [59]. Here $u_{0}$ depends only on $y$ and is positive between the upstream outer walls $y=0,2$. The planar geometry and flow are assumed symmetric about $y=1$ for convenience, the overall pressure differentials as imposed from upstream to downstream are taken to be small, and the outer walls and daughter dividers are all nearly aligned. This is consistent with a multi-branching flow structure comprising an inviscid core in the mother and daughter tubes along with thin $O\left(R e^{-1 / 2}\right)$ viscous layers on the dividers and an $O\left(R e^{-1 / 3}\right)$ viscous outer-wall layer where the divider thicknesses are taken to be of $O\left(R e^{-1 / 3}\right)$.

The core-flow effect is linear, in keeping with the relatively small changes produced by the small pressure differences and near-alignment, so that $u-u_{0}(y)$ is small and equal to $R e^{-1 / 3} \tilde{u}(x, y)$ say with $\tilde{u}$ of $O(1)$. This $\frac{1}{3}$ scaling is due to the divider thicknesses. Hence the small-perturbation system (40) now applies for $\tilde{\psi}$, 

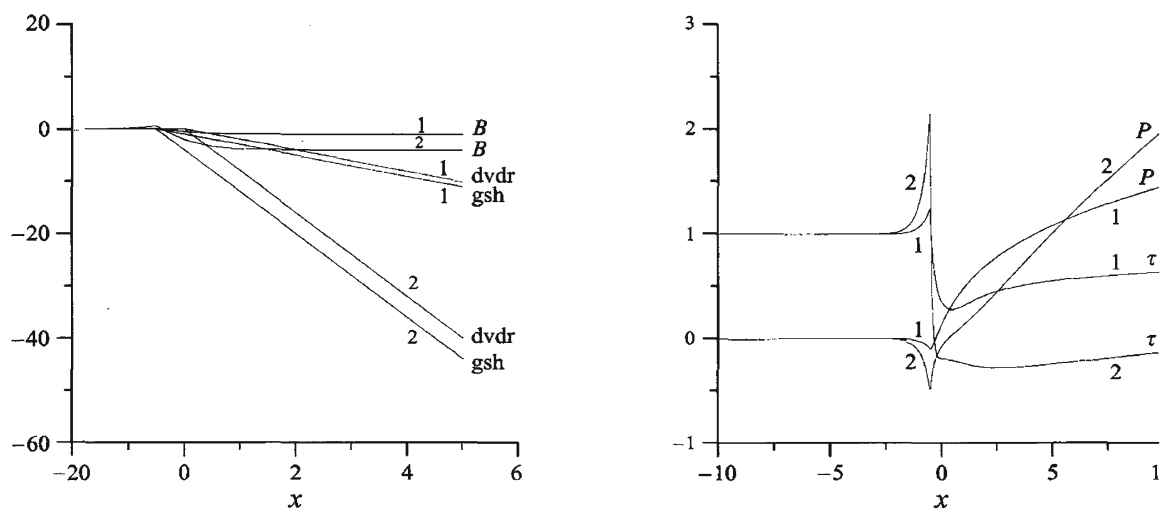

(i)

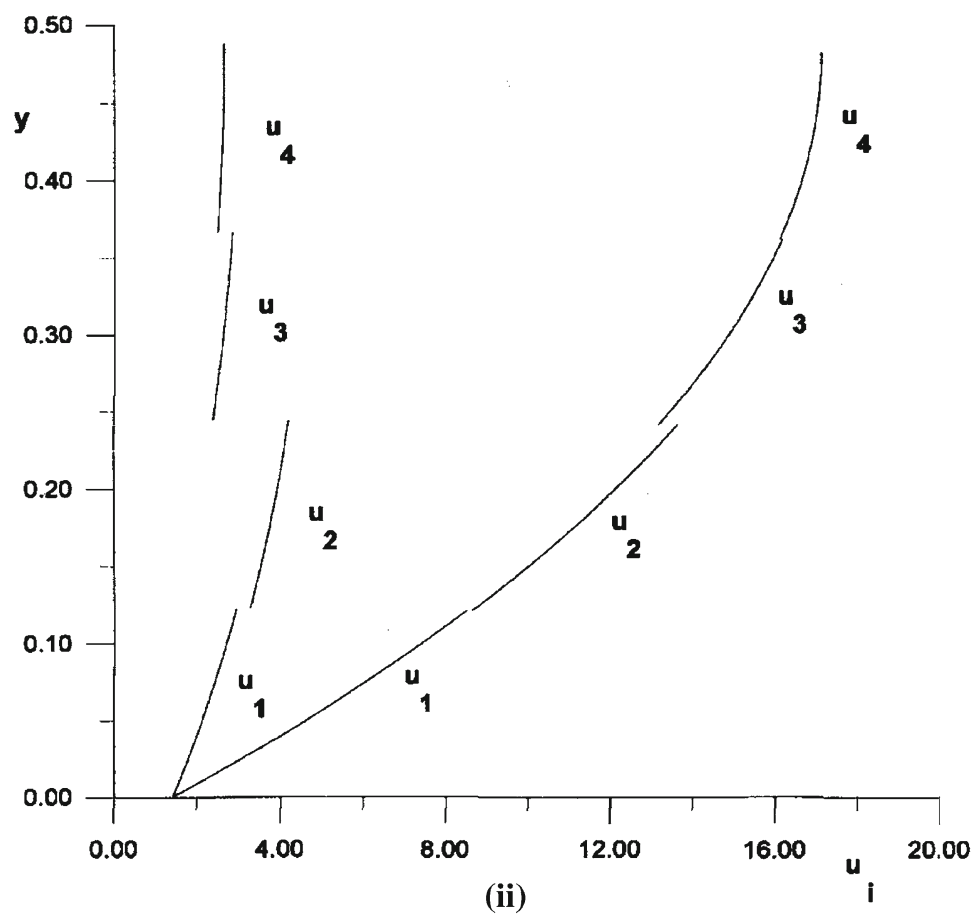

Fig. 4 Branchings over short length scales. (i) Core and viscous wall layer results showing divider shape dvdr, wall shape gsh, interactive effect $B$, pressure and wall shear, for two cases 1,2 . (ii) Dual solutions for the daughter-vessel velocity profiles $u_{i}$, in effect for the branch junction of Fig. 1, given mother and daughter pressures and shapes and $N=8$

where $\tilde{u}=\partial \tilde{\psi} / \partial y$, with the stream function perturbation $\tilde{\psi}$ being zero far upstream and along $y=0,1$ (except possibly for $y=1, x>0$ ). Also, if the divider walls are given by $y=y_{n}+R e^{-1 / 3} f_{n}^{ \pm}(x)$ to $N$, with $y_{n}, f_{n}^{ \pm}(x)$ prescribed, while $c_{n}$ denotes the scaled mass flux value for the $n$ th. divider, then 


$$
\tilde{\psi}=-u_{0}\left(y_{n}\right) f_{n}^{ \pm}(x)+\text { at } y=y_{n} \pm \text {. }
$$

The condition (61) is the tangential-flow constraint. The geometry of the branching is prescribed by the scaled divider shapes $f_{n}$ and scaled outer wall shape $f_{W}$. The internal viscous layers and the outer wall shape $f_{W} \equiv f_{0}^{+}$alike have negligible influence so far. The solution for $\tilde{\psi}(x, y)$ yields a scaled slip velocity $u_{w}$ equal to $\tilde{u}(x, 0)$, i.e., $\partial \tilde{\psi} / \partial y(x, 0)$, at the outer wall.

The outer wall-layer flow is nonlinear (similarly to that in Sect. 3.1). It has $y=R e^{-1 / 3}\left(Y+f_{W}(x)\right)$ and $u, p$ are $R e^{-1 / 3} U, R e^{-2 / 3} P(x)$ to leading order, leaving the boundary layer system $(4,14)$ for $U, \Psi, P$ with $U=\partial \Psi / \partial Y, \partial P / \partial Y$ zero and $R e$ unity. The boundary conditions are as in (36)-(38) except that

$$
U \sim \lambda\{Y+B(x)\} \quad \text { as } \quad Y \rightarrow \infty, \quad B \equiv \lambda^{-1} u_{W}(x)+f_{W}(x)
$$

in view of the matching with the core. As in Sect. 3.1 the system allows separation/flow reversal, if it occurs, to be regular since $P$ is an unknown.

The argument extends to three-dimensional branchings as described in [87], but these induce a logarithmic effect (where any divider meets the outer wall) in the core which limits the applicability of the theory in the present setting: compare Sects. 3, 5.

There are many interesting features. Concerning results, an example is in Fig. 4(i), while [59] focus attention on three specific planar branchings of interest.

- The first is for two large daughters with symmetry about $y=1$, see Fig. 5(i). In this branching the core problem (40) can be solved directly by using a Fourier transform in $x$, to enable the influences of geometric shapes, the incoming velocity profile, the daughter width relative to the mother width, and so on, to be examined. The core solution produces the inviscid slip distributions $u_{W}$ shown in Fig. 5(ii) for several different $u_{0}$ profiles and a given divider shape, a noteworthy feature being the similarity between the distributions. Feeding $u_{W}$ into the viscous walllayer problem via (62) then yields the results for outer wall shear and pressure shown in Fig. 5(iii). These are for several distinct cases 1-7, in each of which the shapes of the central divider $(d v d r)$ and the wall $(g s h)$ are indicated. Cases 1, 2 are examples that have an overall area expansion due to the branching which provokes an adverse pressure gradient and shear reduction downstream, leading in one case to outer-wall separation/flow reversal. Cases 3, 4 have area expansions which are different in geometrical detail, again causing a trend towards flow reversal with a relatively long recirculating eddy downstream, but upstream the effects are opposite to those of cases 1, 2. Cases 5-7 yield an overall contraction of area, which forces a favourable pressure gradient and wall-shear increase downstream.

- The second specific branching is for a small daughter as shown in Fig. 6(i), where $\alpha$ is the daughter width scaled relative to the mother width. The core solution yields the results for $u_{W} / c_{1}$ presented in Fig. 6(ii) as $\alpha$ is varied. The results confirm in particular that most of the change in $u_{W}$ occurs within a short distance $O(\alpha)$ of the daughter mouth when $\alpha$ is small, e.g. 0.1 or less, the other distinguished length scale being $O(1)$ ahead of and after the mouth. Over the latter global scale the full profile $u_{0}$ has effect but the daughter acts as a sink-like disturbance at the wall, 


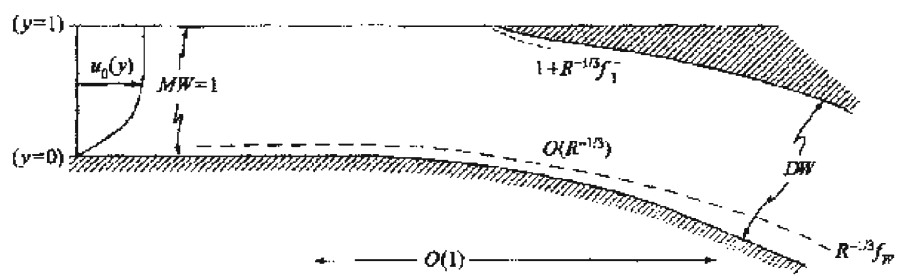

(i)

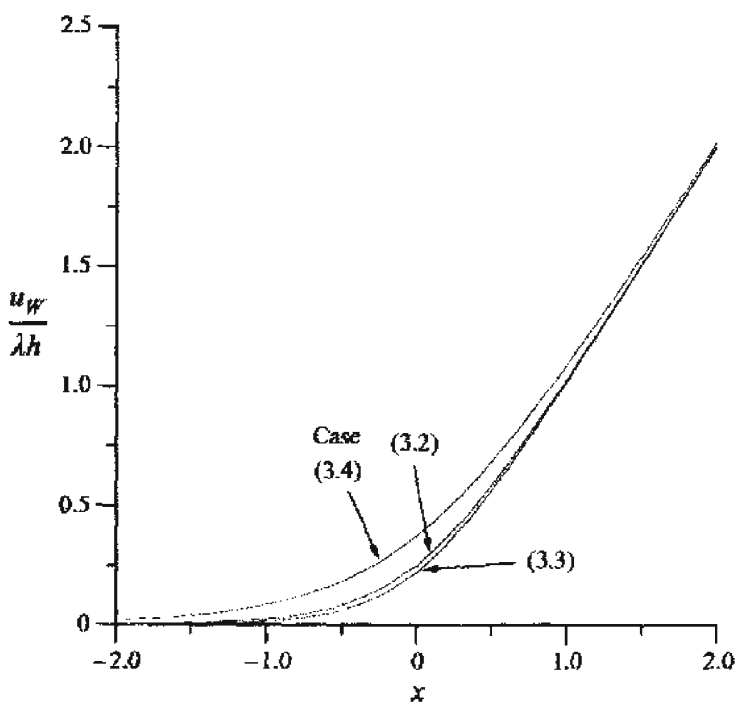

(ii)

Fig. 5 i Symmetric branching flow: MW is the width of the mother-vessel, DW is that of the daughter. ii Scaled slip velocity (contributing to the negative displacement effect) induced at outer wall in three cases, versus $x$. iii Further cases, showing the induced scaled displacement, pressure and shear stress at the outer wall

whereas over the $O(\alpha)$ local scale the details of the daughter mouth are apparent and only the incident shear flow $\lambda y$ drives the local flow. Properties in this specific branching prompt the work in Sect. 5.1.

- Third is the branching for many small daughters as illustrated in Fig. 7(i), where $N \gg 1$ but $\alpha \ll 1$. Now the solution in the inviscid core gives in detail the $u_{W}$ results in Fig. 7(ii) as $N$ increases: $u_{W}$ is plotted instead of $u_{W} / c_{1}$ for clarity. Here again length scales of orders unity and $\alpha$ operate. The mother flow ahead of the multi-branching at $x=0$ poses a half-range problem in which the pressure at $x=0-$ is given, being prescribed by the individual daughter flows each of which tends to act alone; between them is the $O(\alpha)$ region at each daughter mouth. Associated wall-layer features are shown in [59], indicating in particular that this multi-branching can permit enhanced turning of the overall flow without significant flow reversal. The findings prompt the study in Sect. 4.4. 


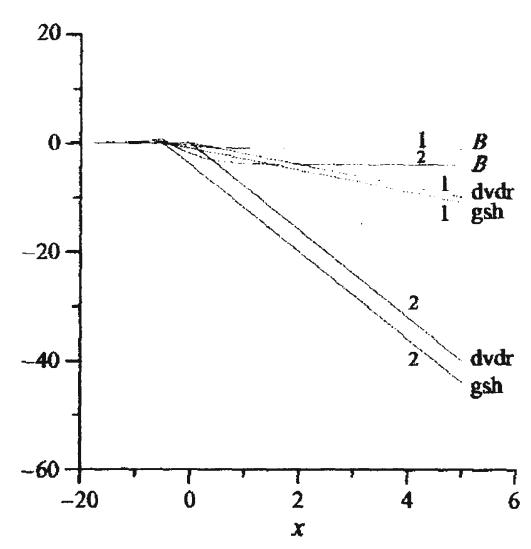

(a)
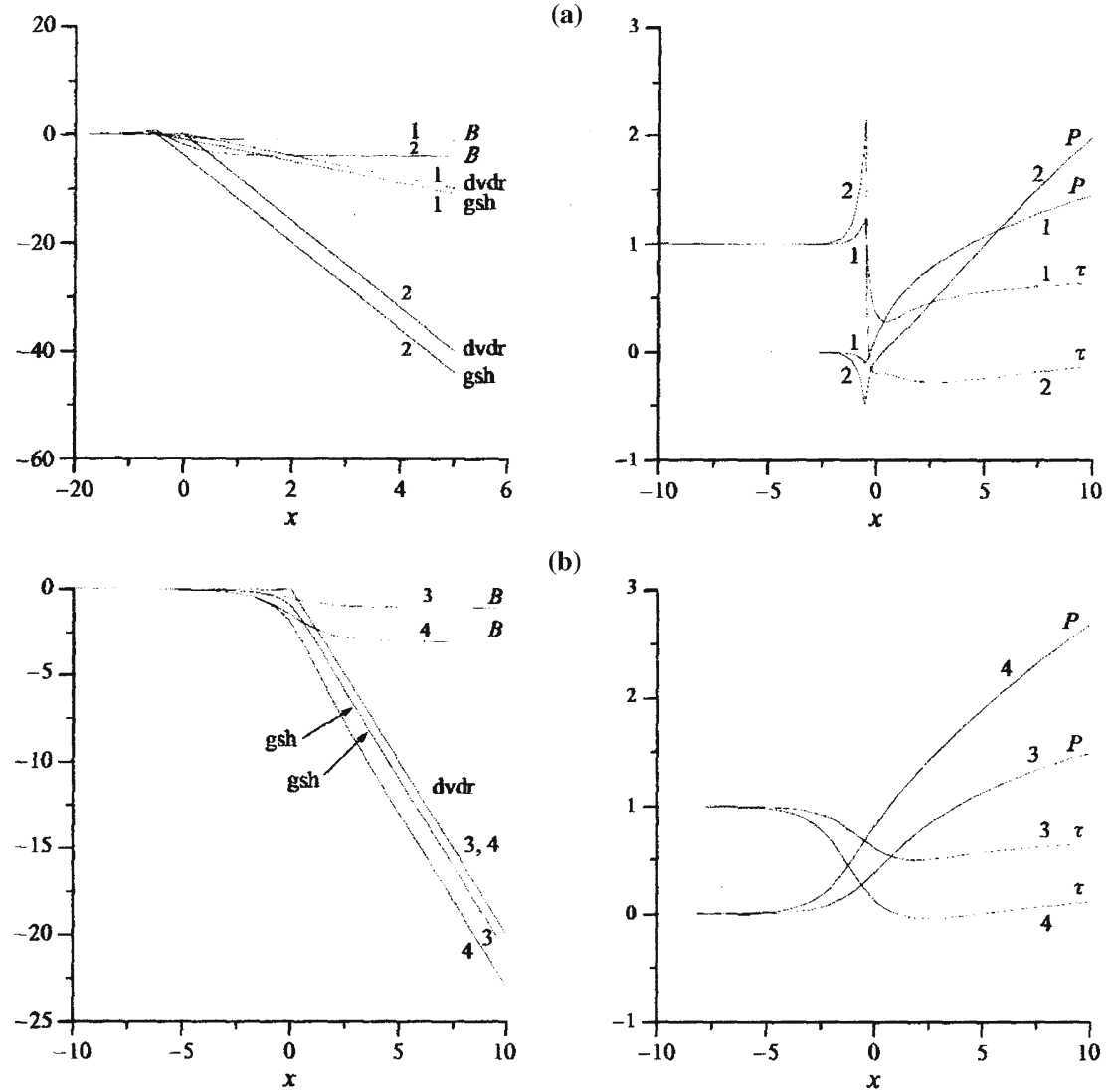

(b)

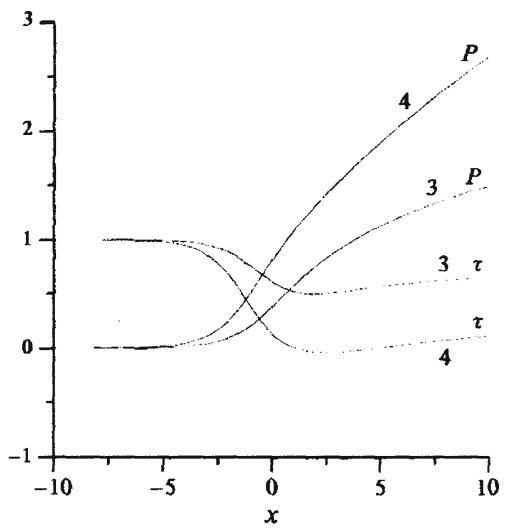

(c)
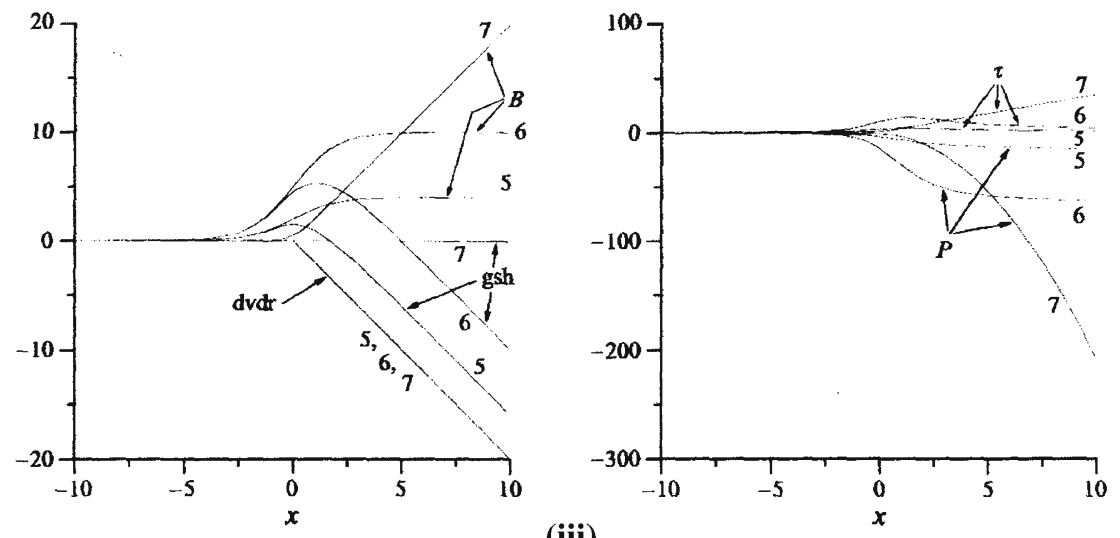

(iii)

Fig. 5 continued 


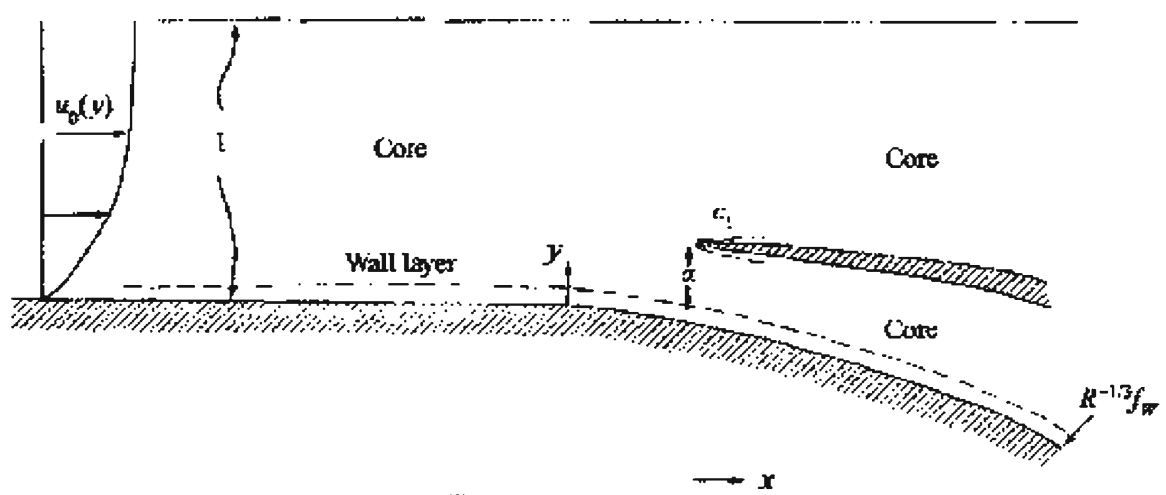

(i)
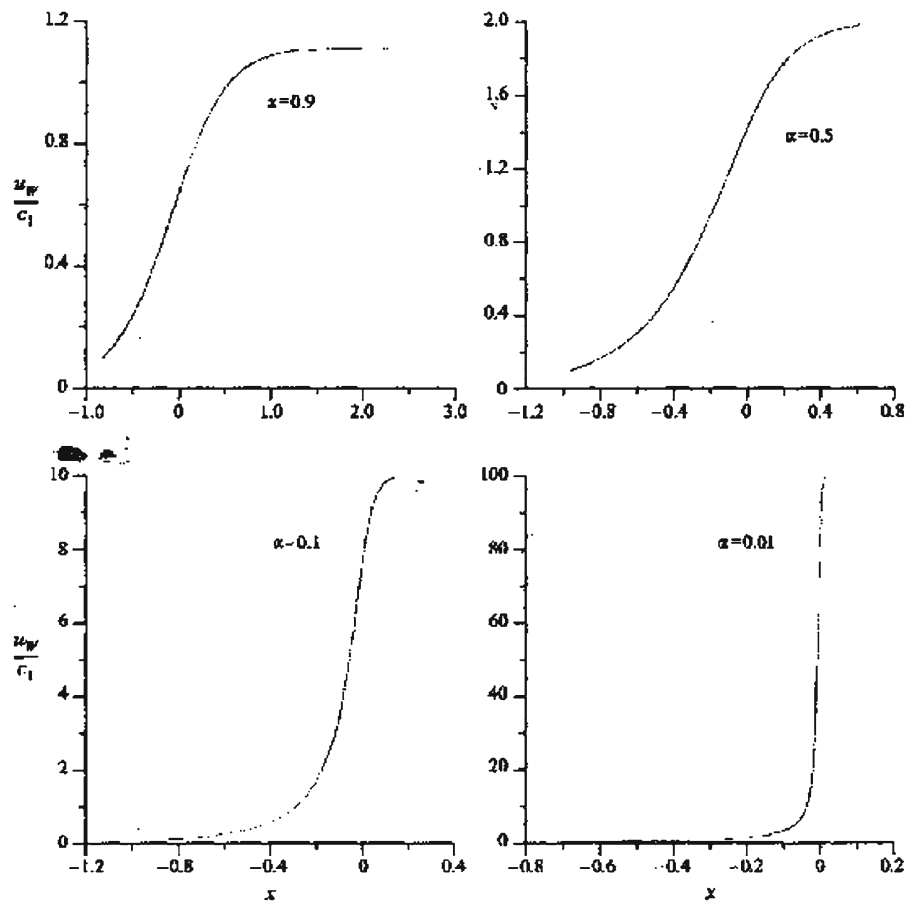

(ii)

Fig. 6 i With a divider near an outer wall. ii Outer-wall slip velocities or negative displacements. See [59]

In addition, qualitative comparisons can be made with the direct numerical simulations of the Navier-Stokes equations in [87] over their Re-range 200 to 1200, for a fixed geometry with typical turning angle $\alpha=\pi / 6$ at successive wedge-like branchings. At $R e=200,500$ confined recirculatory eddies are found in their Fig. 3 at most of the outer walls after each branching, and the eddy lengths increase with increasing $R e$. In contrast, the wall shear is enhanced both on the outer wall ahead of the branching 


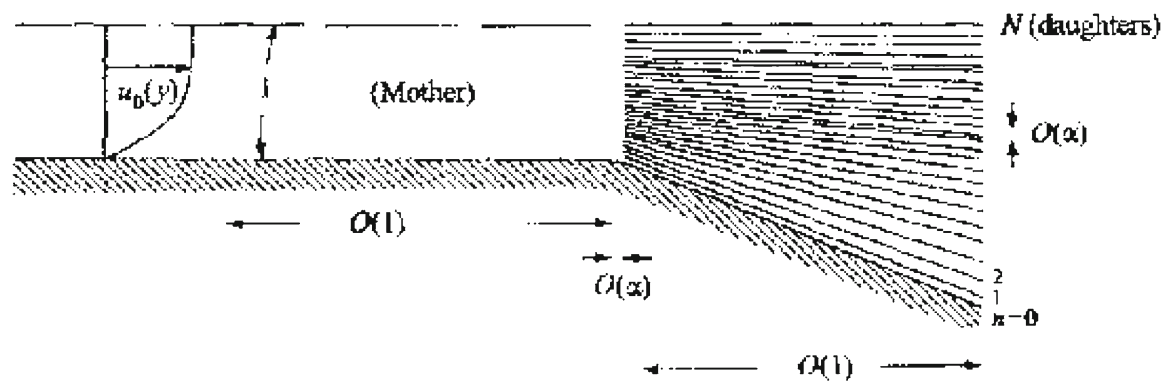

(i)
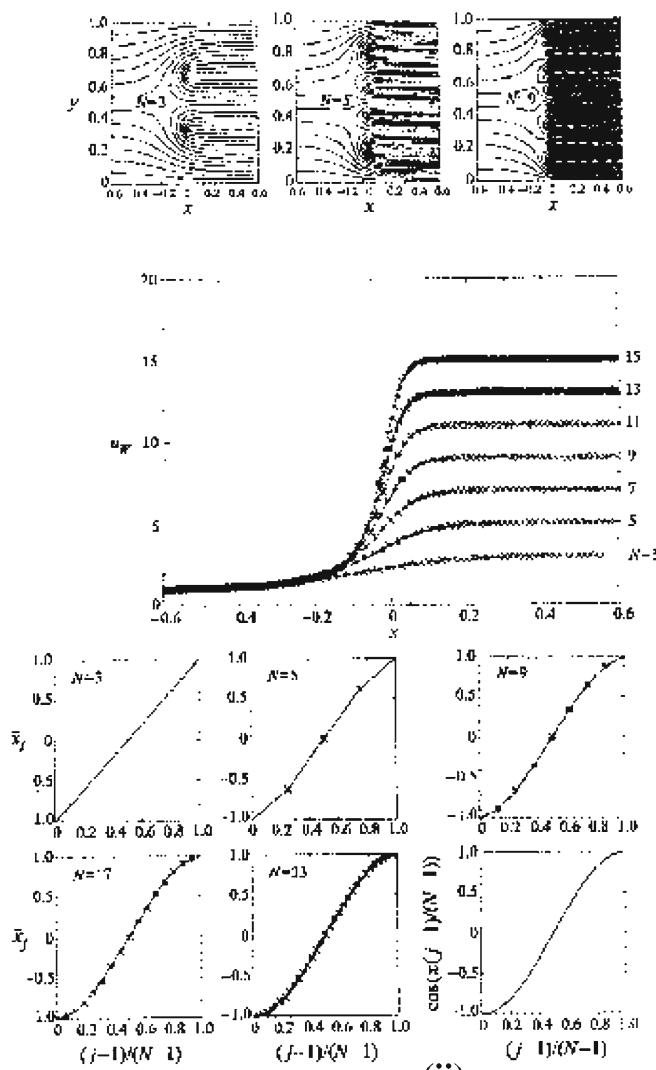

(ii)

Fig. 7 i With many daughter vessels. ii Streamlines produced in the core motion and related solution properties. See [59]

and on the inner divider wall. The reversed-flow trend is such that at $R e=900,1200$ one outer-wall eddy extends downstream to the next branching and joins with the next outer-wall eddy, while others continue to elongate. (A new eddy also forms at $R e$ of 
1200). These features of the simulations are supportive of the theory for branching as in the first bullet point above which does indeed predict increased outer-wall flow reversal as $R e$ increases; see also ([87], figure 4). The theory further predicts (e.g. see Fig. 5(iii)) enhanced wall shear upstream of branching and on the dividers, as in the simulations [87]. Moreover the extra turning produced by successive branchings in [87] without excessive reversals arising also is in keeping with the theory for the third bullet point above. The comparative smallness of the critical $R e$ for flow reversal, about 100 , for an $\alpha$ value of $\pi / 6$ in [87] is not inconsistent with the predicted $O\left(R e^{-1 / 3}\right)$ scale for $\alpha$. Further comments and comparisons are presented in Sect. 4.5.

\subsection{Substantial changes in cross section}

In this kind of multi-branching the scaled pressure differentials are $O(1)$ and the local geometry is not necessarily slender, by contrast with the branching flows studied in Sect. 4.3. This account again builds on length scales described in Sect. 2.2 but the focus is on the local scale (56).

The theory here based on that in [88] is for planar flow in the branching geometry of Fig. 1. In nondimensional terms, a single mother tube, of width 1 and containing fully developed incident flow of unknown total mass flux $\lambda$, branches locally into $N$ daughter tubes of total width $\bar{A}$ ('area') at large positive $x$; in the figure $N$ is 5 . The exit velocities $u_{1}$ to $u_{N}$ in the daughters are unknown. The branching shape involves arbitrary $O(1)$ slopes and is prescribed, as are the daughter pressures $\pi_{1}$ to $\pi_{N}$ downstream, which are measured relative to the upstream mother pressure. The latter is taken as zero. The orders of magnitude point to an inviscid response $(4,12)$ in the absence of significant separation.

Conservation of mass and pressure head apply in each daughter, effectively as in (27-29), to determine $\lambda$ under various area and pressure settings by means of a set of nonlinear recurrence relations and ordinary differential equations, with all the $u_{i}$ for $i=1$ to $N$ being assumed positive. One significant overall property comes from using an integration of $d y=d \psi / u$ in effect, incorporating (27-29) indirectly and summing over all the daughter tubes downstream. In consequence the overall problem takes the form

$$
\bar{A}=\sum_{i=1}^{N} \int_{\psi_{i}^{-}}^{\psi_{i}^{+}} \frac{d \psi}{\left\{\psi_{0}^{\prime}\left(\psi_{0}^{-1}(\psi)\right)^{2}-2 \pi_{i}\right\}^{1 / 2}}
$$

where the size of the incident stream-function profile $\psi_{0}$ is to be found (i.e. the factor $\lambda$ is to be determined) together with the stream-function values $\psi_{\underline{i}}^{ \pm}$on the walls of each daughter. The calculated response of $\lambda$ as the prescribed area $\bar{A}$ is varied is given in Fig. 8(i) for two types of pressure settings. The first has all the pressures $\pi_{i}$ being negative, in which case a unique non-separated flow is predicted for any $N$ throughout the interval $0<\bar{A}<1$; the figure shows results for a range of $N$ values along with asymptotes labelled I-III which stem from (4.1) for $\bar{A} \rightarrow 0, \bar{A} \rightarrow 1, N \rightarrow \infty$ 
Fig. 8 Multi-branching [88]. i Mass flux versus flow area in two major scenarios; the second includes dual solutions. ii The dependence of the mass flux on the number $\mathrm{N}$ of daughter vessels, in four cases

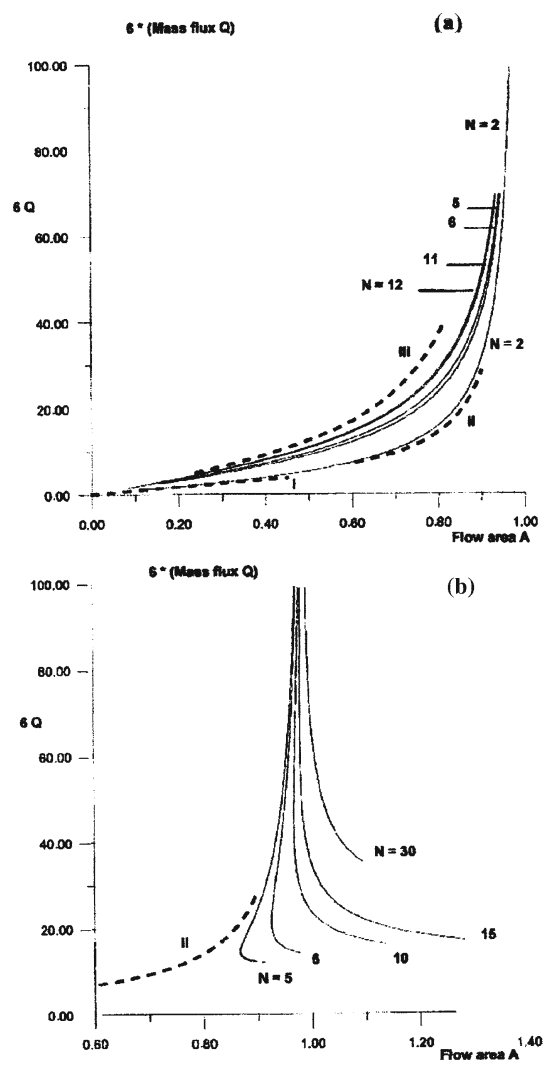

(i)

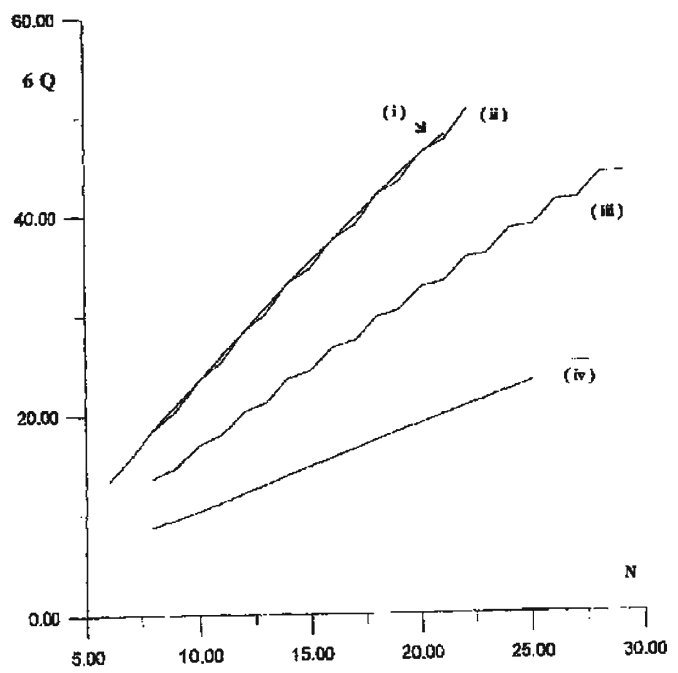

(ii) 
respectively and provide a comparison. This type allows no solution if $\bar{A}$ is unity since the implied increase in $u^{2}$ and hence $u$ is then incompatible with the total mass-flux requirement. The second type has at least one $\pi_{i}$ being positive and that leads to restrictions on the left and right as non-separated flow is found to be impossible if $\bar{A}$ is too small or too large; non-uniqueness can also arise then, as indicated in our Fig. 4(ii) which demonstrates the notably different flow profiles predicted downstream. Concerning the restrictions above, in practice the flow may separate substantially or the velocity $u_{i}$ may be reversed for some $i$ values, negating the above analysis: see direct simulations below and the comments at the end of the paper. Figure 8(ii) shows the dependence of $\lambda$ on $N$ for given pressure settings all of the second type and given area $\bar{A}$, in four cases (i)-(iv). A remarkable feature is the linear increase of the total flux $\lambda$ with increasing number of daughters $N$, in every case, for $N$ above about 4 . This and the asymptotes mentioned previously are analyzed in [88].

In broader terms, for instance in a complete network model, the $\pi_{i}$ values themselves are governed by interaction between the shorter-scale inviscid problem above and the viscous development over the longer $O(R e)$ length scale (Sect. 2.2). This feedback is akin to that in the side-branching studied in Sect. 5.1, and [88] investigates one example of it.

\subsection{Direct simulations and comparisons}

Direct numerical simulations are included here, along with comparisons and comments on "steal" for example. There is fair agreement as $R e$ increases.

Near a multi-branching junction, the flow response over streamwise lengths (56) is expected to matter most when $R e$ is medium to large. To test this expectation, we first describe a numerical investigation of the full viscous problem (4-7) for such a junction. The system is subject to the boundary conditions of no slip at all the fixed solid surfaces and of unidirectional flow sufficiently far upstream. Thus in particular

$$
\begin{aligned}
& u_{x}=0, \quad v=0, \quad p=p_{-\infty} \quad \text { at } x=x_{-\infty}, \\
& u_{x}=0, \quad v=0, \quad p=p_{\infty} \text { in the } N \text { daughters at } x=x_{\infty} .
\end{aligned}
$$

Here $x_{-\infty}, x_{\infty}$ are the end station values, suitably far upstream and downstream respectively, while the zeros of $\partial u / \partial x, v$ in $(64,65)$ correspond strictly to those stations being infinitely far upstream and downstream. This allows necessary flexibility regarding the inflow and outflow, although near-Poiseuille flow is generally found to emerge anyway in the results at medium $R e$ values. The values $p_{-\infty}, p_{\infty}$ are the prescribed end pressures upstream and downstream, $N$ is the number of daughter channels, and the walls of the mother channel upstream are given by $y= \pm 1$, for $x<0$. The branching itself occurs at $x=0$. In each daughter, where $x>0$, the coordinate $y$ is again defined as perpendicular to the daughter wall. The mass flux in total through the mother and through the daughters is unknown. The branching geometry is to allow for expansions and contractions but by means of straight sections of channel. The branching flow was treated by a direct finite difference method [60]. The end stations were usually taken at $-1,1$ as fundamental cases. Reduced downstream areas were obtained (i.e. $A<1$ ) 


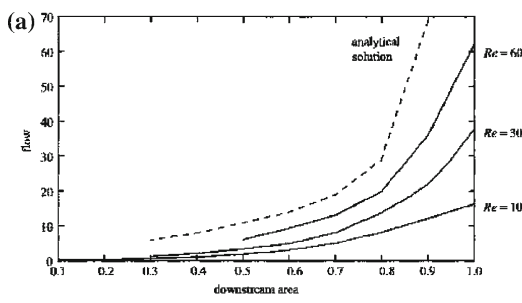

(c)
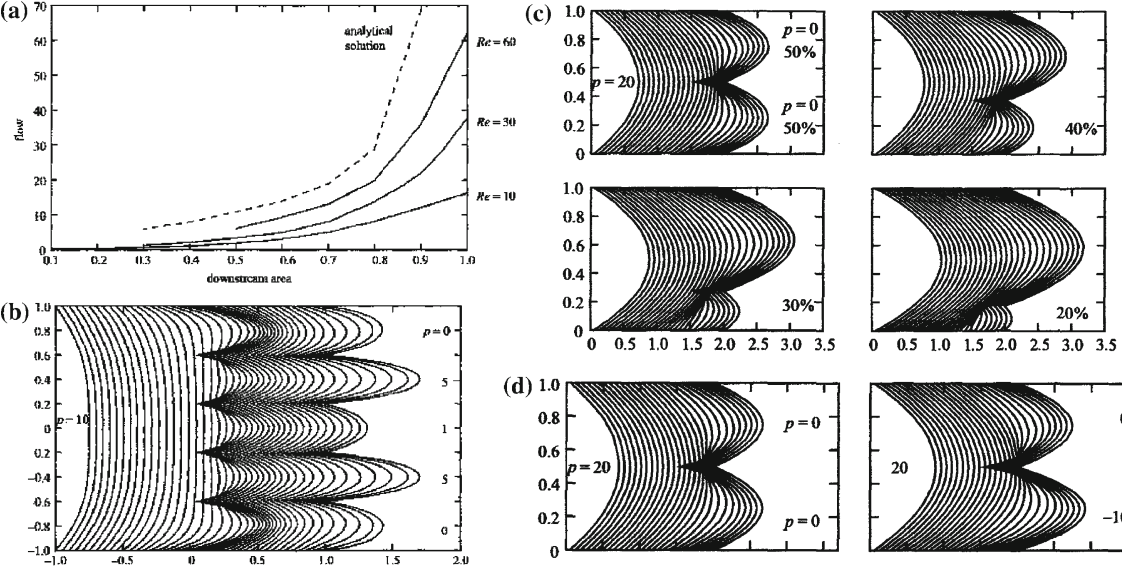

(d)
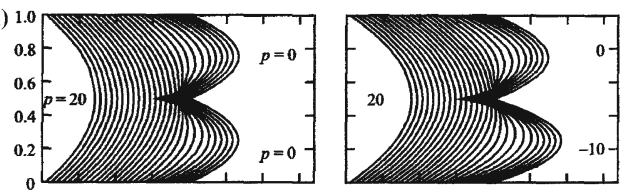

(i, ii)
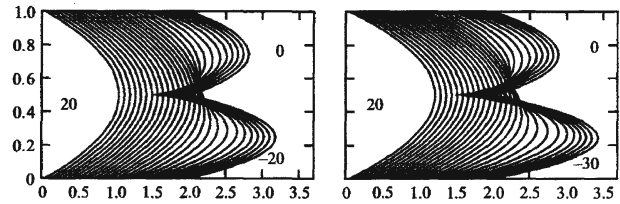

(e)
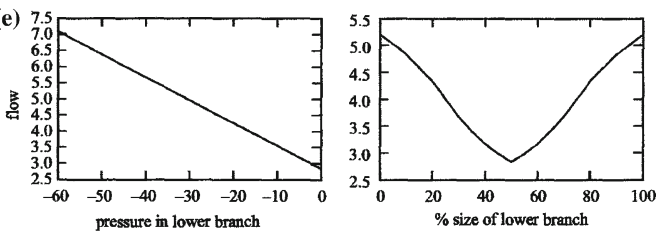

(iii-v)

Fig. 9 Directly simulated branching flow. (i), (ii) For varying downstream areas and Reynolds numbers, along with a comparison with mathematical analysis. (iii)-(v) The effects of geometry and pressure settings on the branching flows

by closing off some of the downstream area nearest the outer wall, effectively shutting off a daughter tube.

Solutions are presented in Fig. 9(i-v). A typical case required around 500 iterations to converge to within a small tolerance. As $R e$ increased, substantial under-relaxation was necessary to achieve a converged solution. The grid size was generally taken to be 0.05 in both directions, but refinement was used to ensure accuracy of the solution. To help resolve the thin wall layers present, the results below $R e=200$ have a grid size refined to 0.02 , although they are virtually identical to those from the coarser mesh in terms of velocity profiles and through flow.

The results can be compared with the limit analysis of Sect. 4.4. Thus Fig. 9(i) compares the through flow generated over a range of downstream areas $A$ as predicted by analysis [88] and by the current finite $R e$ method. It shows the case $N=11$ for $R e$ ranging from 10 to 60 . The comparisons indicate fair agreement as $R e$ increases. Further comparisons are presented in [50], including cases where flow is forced down a daughter tube against the pressure gradient; that is, with one daughter pressure higher than the upstream pressure while the other daughters have a low pressure. Forward 
flow is obtained in all daughters in some cases. This phenomenon is also found in the inviscid theory of Sect. 4.4. Figure 9(ii) shows velocity profiles obtained at $R e=70$ for a case with $N=5$. Figure 9(iii-v) present results for a mother splitting into just two daughter branches at $R e=200$. Figure 9(iii) shows the $u$ velocity profiles for various divider positions with downstream pressures being equal in each daughter. At these medium Reynolds numbers near Poiseuille flow emerges at the inlet and, after some deformation as the branch splits, the solution appears to settle back into near Poiseuille flow very quickly after the bifurcation. We note here that such a fully developed motion upstream and downstream is not assumed a priori; it emerges at these $R e$ values. At large $R e$, the downstream influence length or entry length has the well-known long scale $O(R e)$ as in Sect. 2.2, whereas the upstream influence length is usually of order $R e^{1 / 7}$ as in Sect. 6 , consistent with the present numerical results. Figure 9(iv) provides the $u$ profiles with equal downstream sizes but varying the lower daughter pressure from $p=0$ (the symmetric case) to $p=-30$. As might be expected, when $p$ is decreased the flow through that daughter, and the entire system, is increased. Finally, Fig. 9(v) displays the through flow in each case. For the varying area ratios, the nearer to the symmetric case the less through flow is generated for a given pressure distribution. In the varying pressure case, the through flow increases seemingly linearly with the magnitude of the overall pressure drop.

\section{Branchings with viscous-inviscid or long-short interactions}

The coming sub-sections on two-dimensional branchings cover examples where interaction may play a substantial role, either via a balance between viscous and inertial effects or via feedback between long and short length scales. These are summarised in Fig. 10 (i,ii) and concern a side-branch in Sect. 5.1, larger networks in Sect. 5.2 and then wall layers in Sect. 5.3, leading to many interesting features.

\subsection{Side-branching}

The axial scale

$$
|x| \sim 1
$$

is again active here, with the thickness scale being that in (30). The wall-layer system $(4,12)$ applies in the mother vessel close to its wall and in the side branch which is a small daughter vessel. A novel feature however is due to the action of "jump" effects which arise over a shorter length scale

$$
|x| \sim R e^{-1 / 3}
$$

and these provoke long-scale/short-scale interaction effectively, as in Fig. 5, together with an interesting balance of Euler forces $(4,8)$ and lubrication forces $(4,13,21)$ in certain situations associated with high decay rates in the axial direction. 

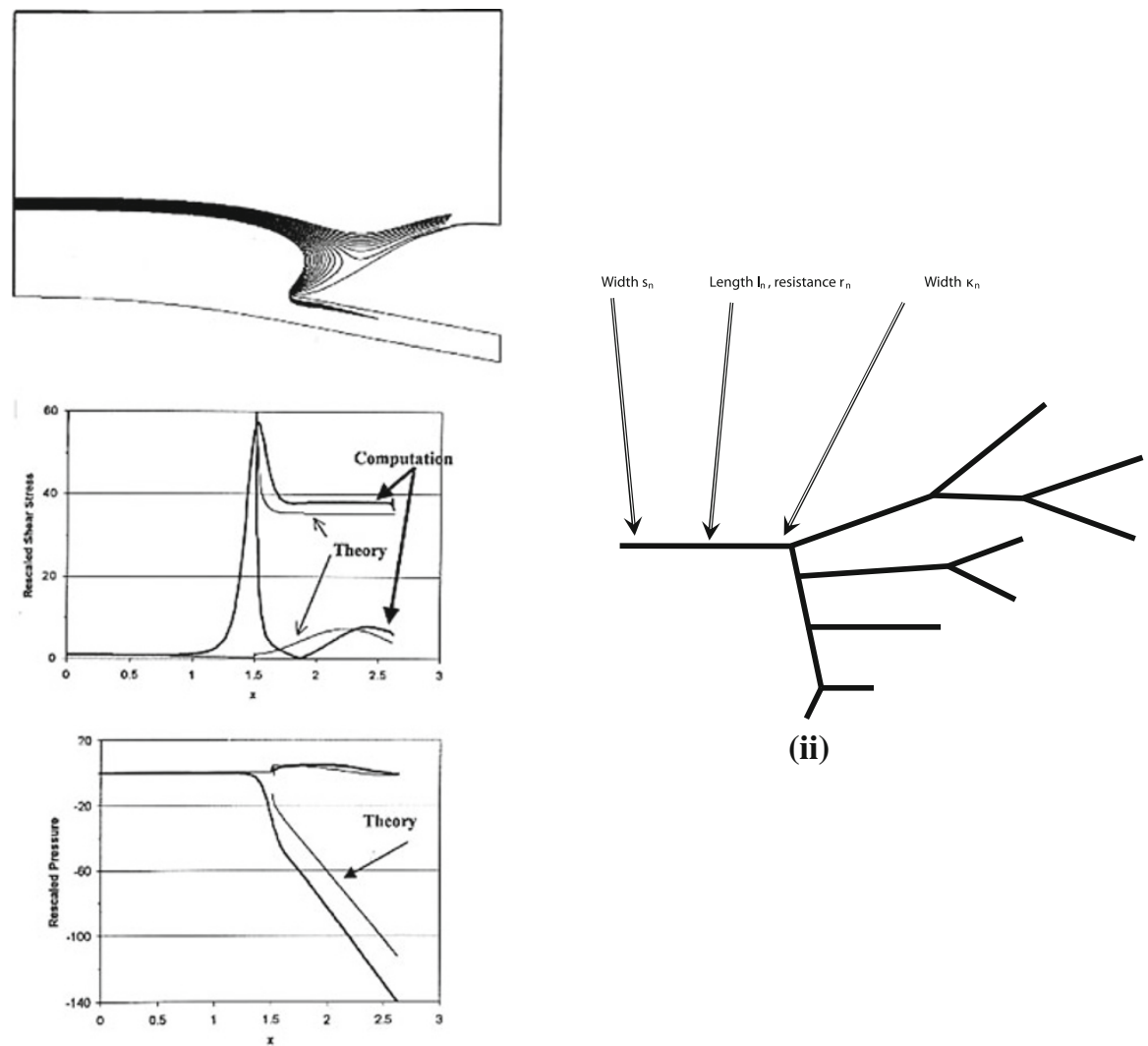

(ii)

(i)

Fig. 10 Branchings with interaction. i For a side-branching: sample streamlines in the mother, some of which enter the side-branch daughter vessel; wall shear stress along outer (lower) wall from present theory and from direct numerical computation; wall pressure from theory and direct computation. ii For the enlarged network of Sect. 5.2

The study here of slender side-branching stems from the special case (ii) in Sect. 4.3 and again has potentially wide generic application. The work is based on [61] and connects with that in Sects. 3, 4. Now however the pressure differences imposed are taken to be relatively substantial and the size of the small daughter or side-branch is reduced such that it all lies within the viscous near-wall layer as in our Fig. 10(i). The essential difference in modelling between smaller and more substantial pressure changes, in the present setting, is that here a viscous-inviscid balance of effects describes the motion almost everywhere. Figure 11(i) shows a three-dimensional side branch, while Fig. 11(ii) shows the two-dimensional case, with the mother-flow regions (i), (ii), in the present nondimensional form based on the incident shear and the main streamwise length scale of the branching. The incident mother shear flow is uniform because of the near-wall position and size of daughter. The imposed pressure within the daughter at some position $x=x_{2}$ downstream of the daughter entrance $x_{1}$ is denoted by $P_{\infty}$, the mother pressure upstream being taken as zero, and $f_{1}-f_{4}$ are the known 


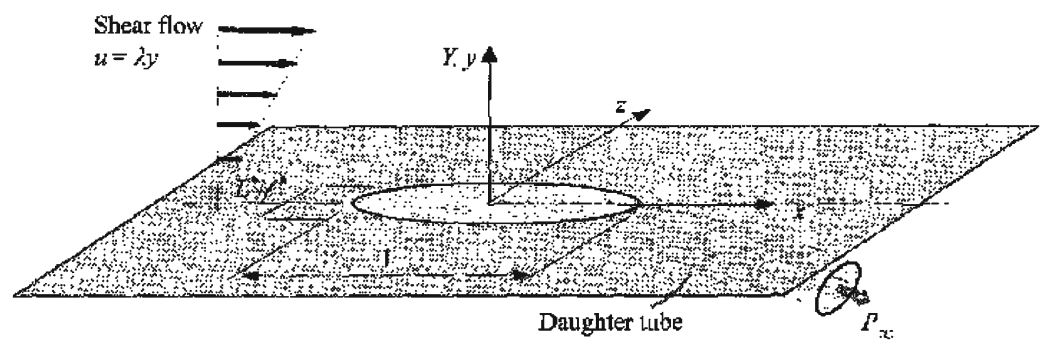

(i)

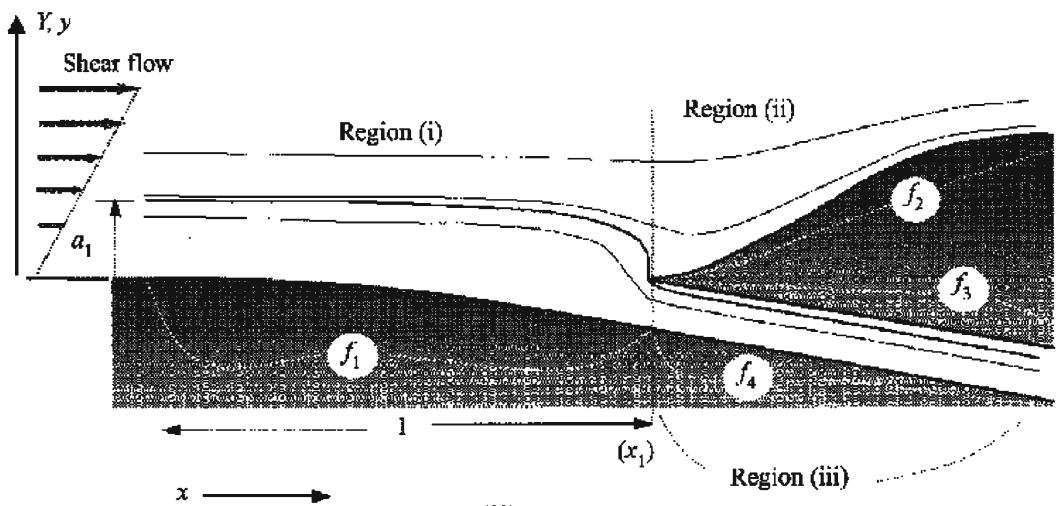

(ii)

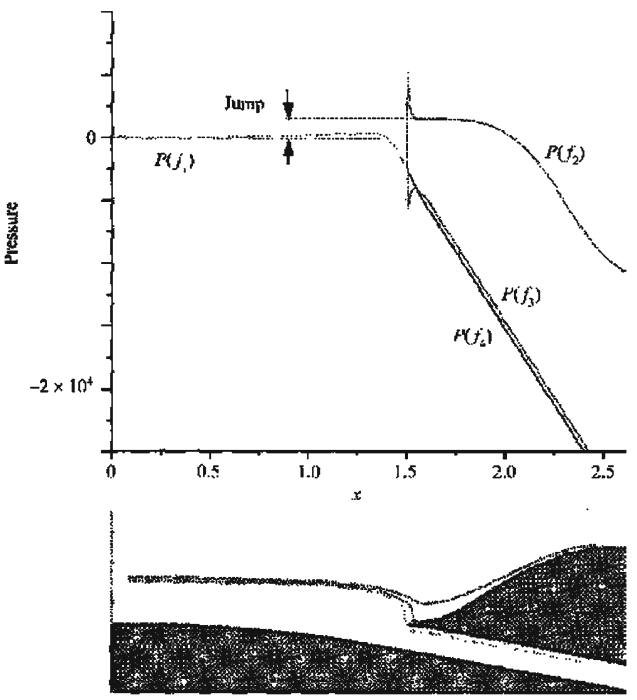

(iii)

Fig. 11 With a small side-branch [61]. i Three-dimensional version. ii Two-dimensional version, including the various regions and shape functions. iii Direct simulation results for pressure and streamlines 
normalized wall shapes, again in coordinates $x$ and normalized $y$ or $Y$, whereas the scaled thickness $a_{1}$ of mother fluid that is entrained into the daughter is unknown. The constants $\lambda, L^{*} / l^{*}$ denote the normalized shear and a ratio of the daughter-mouth dimensions respectively. Figure 11(iii) presents a result from a direct simulation at a moderate value of $R e$, showing the wall pressures. Most noteworthy is the apparent jump or rapid spatial change in pressure close to the mouth of the side-branch.

The explanation for the jump is as follows. For sufficiently large $R e$ the slender-layer equations $(4,12)$ hold almost everywhere, subject to standard boundary conditions of no slip at each wall, and these suggest parabolic dependence in the direction forward from the uniform-shear condition given upstream. Yet such a parabolic dependence on its own must usually violate the required downstream condition

$$
P^{-}=P_{\infty} \quad \text { at } \quad x=x_{2}
$$

in the side branch, in which the pressure is $P^{-}$. The only resolution for this is that a discontinuity (jump) has to be present, specifically at the mouth $x=x_{1} \pm$, achieved by means of a local essentially inviscid Euler zone as in $(4,8)$ which conserves mass and pressure head as in (27-29), so that

$$
\Psi \text { and } P+\frac{1}{2} U^{2} \text { are conserved }
$$

along streamlines; then $P, U^{2}$ individually can jump across the zone $\left(x \rightarrow x_{1} \pm\right)$. The jump here is supported by the local configuration of the surfaces $f_{1}, f_{3}, f_{4}$ combined with the incident shear. A jump cannot be sustained across any station $x$ other than $x_{1}$.

Again there are interesting features to focus on. The system $(4,14)$ incorporating $(68,69)$, and thus predicting jumps in $P$ and wall shear $\tau_{W}$ or $\tau$, was solved numerically in two and three dimensions by rapid forward marching, sample results being given in Figs. 12(i-iii) for steady planar motion. Also presented are analytical results for comparison. Figure 12(ii) of then shows comparisons with direct simulation results for pressure and wall shear at moderate $R e$ for an individual case, while Fig. 12(iii) covers a range of cases (corresponding to different entrainment values) according to the model and compares with the direct simulation values obtained for two of those cases; see also our Fig. 5(i). The agreement is encouraging throughout.

The study in [61] additionally includes unsteady, three-dimensional and higher suction effects. This last effect provokes a sink-like behaviour at the branch mouth, the sink strength increasing with $P_{\infty}$ and hence with $a_{1}$ and gradually generating substantial upstream influence. The influence is favourable, in the sense of reducing or suppressing any upstream separation. Downstream separation allows some fluid particles to flow past the side-branch before being dragged back into it.

\subsection{Larger networks}

The second configuration to be addressed in this section concerns the large network analysis of [60] for successive bifurcations from one mother to two daughters, four grand-daughters and so on. The two principal length scales axially now are 

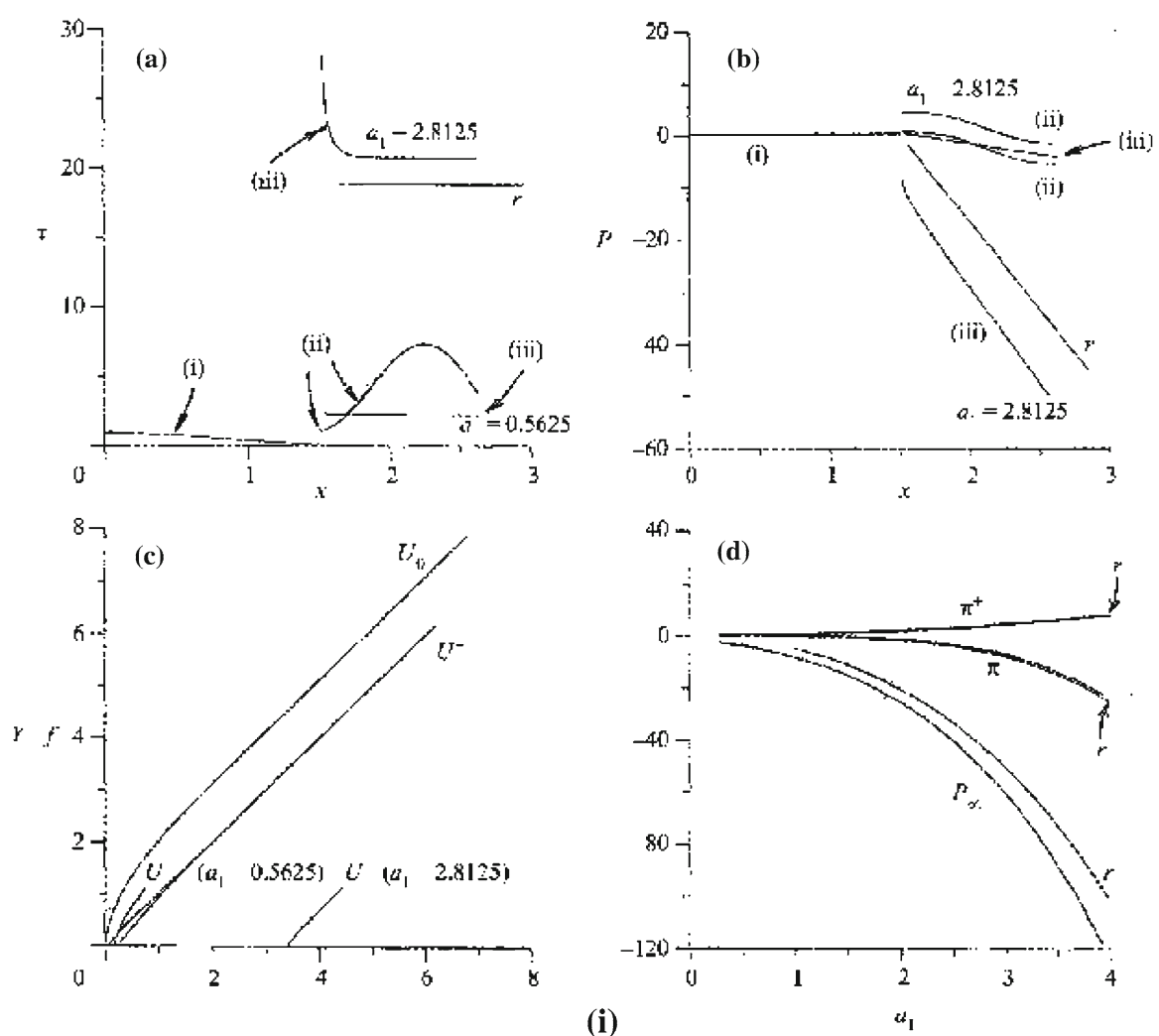

Fig. 12 With a small side-branch [61]. (i)-(iii) Theoretical/analytical results in various cases and comparisons with direct simulations

$$
|x| \sim 1 \text { and }|x| \sim R e .
$$

The approach involves units which comprise the comparatively thin vessels acting in essence over a long viscous scale and hence governed by $(4,12,13)$ or $(4,13,21)$, joined together by means of short-scale junctions where inviscid responses similar to $(28,29)$ are appropriate.

For the network (Fig. 10(ii)), we presume the pressure drop between the original entrance and the end vessels furthest downstream is known, as well as the details of all vessels (resistance and diameter, etc.), and we seek the total flux. The flux and pressure drop through individual vessels can then be calculated. The generic model makes some central assumptions. First, the flow is planar. Second, a long-short scale split is exploited. The dynamics in individual vessels are over such a long scale that fluid inertia may be neglected, so that a fully developed Poiseuille profile (26) holds. Hence, the pressure drop over a vessel is proportional to the flux through it, or to the mean velocity at its entrance. In a sense, that is the far-field view.

In contrast, the flow locally at the junctions (i.e. in the near-field) is so spatially rapid as to be governed by inviscid dynamics as in Sect. 4.4. Here vorticity and pressure head 

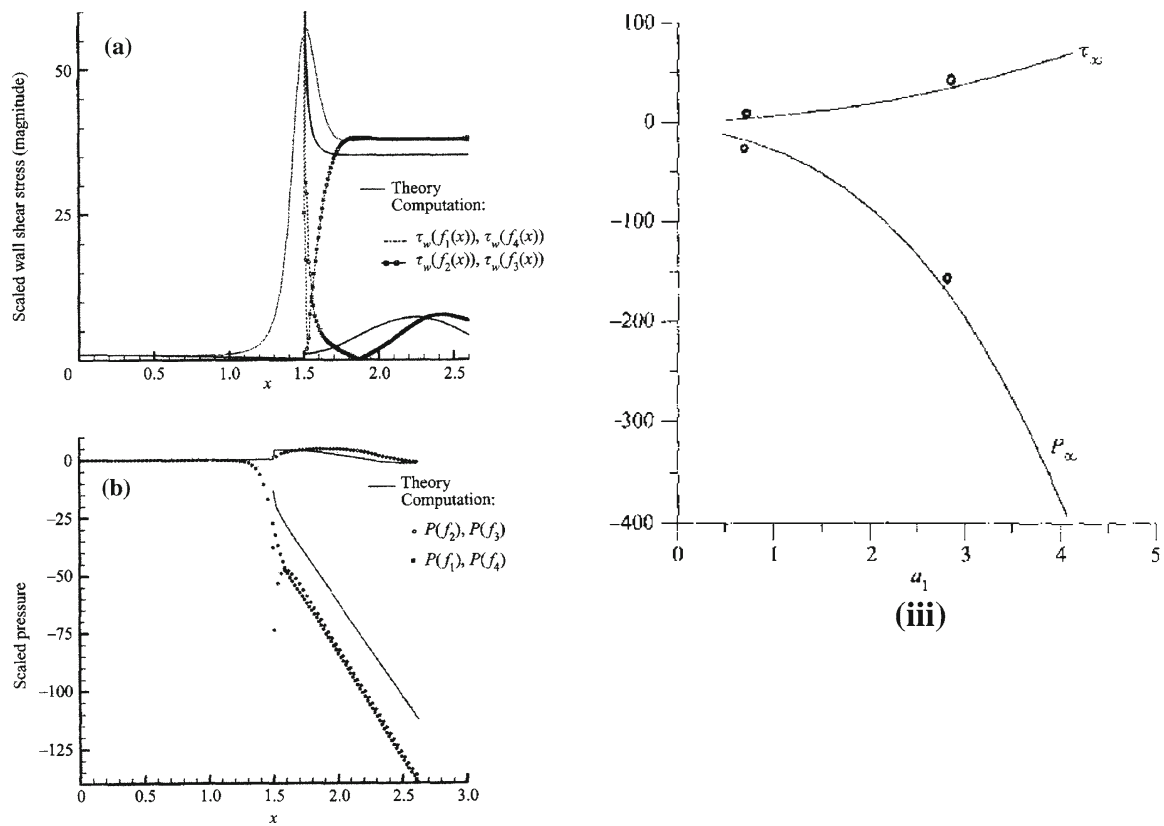

(iii)

(ii)

Fig. 12 continued

are conserved along a streamline, which with mass conservation allows calculation of the downstream velocity profiles along with nonlinear expressions for the pressure drops suffered on passing into the downstream vessels. These profiles then develop into the Poiseuille profile (26) owing to viscous action on an intermediate scale that is short compared with the present vessel length as noted in Sect. 5.1.

The short-long dynamics are combined through the global condition of given total pressure drop from the original mother to any end vessel, giving a nonlinear system for the flux in every vessel. In principle, this system is solvable but here, in an attempt to understand the entire network, we make a third assumption of replacing the incoming Poiseuille profile at a given branch by a uniform plug flow with the same mass flux. This assumption approximates the second above as [88] in Sect. 4.4 shows that it emerges naturally for increasingly large numbers of downstream branches for $N$ above approximately three. Additional comparisons show that this simplification gives results similar to those from the full equations in cases where the flow remains forward and, equally important, is asymptotically correct in the limit of an equal division of the oncoming mass flux into the downstream vessels. In addition, we restrict consideration to junctions with net cross-sectional area decrease to prevent separation. Nevertheless, area increase can be accommodated here by means of the longer-scale (far-field) geometry, where the model allows slow variation in an individual vessel width over its length when the flow remains attached, thereby accommodating networks where the net area increases. 
The network thus consists of a concatenation of units (Fig. 10(ii)), with a single parent vessel upstream. A unit has the downstream end of that parent vessel, splitting into two over a short scale, and the full extent of the two downstream vessels. A vessel identified by subscript $n$ (with the original mother having $n=1$ ) has upstream width $s_{n}$ and downstream width $\kappa_{n}$. The pressure drop along its length (effectively in the far-field) is $U_{n} r_{n}$ say, where $U_{n}$ is the cross-sectionally averaged velocity at the upstream end and

$$
r_{n}=\frac{12 s_{n}(0)}{\operatorname{Re}} \int_{0}^{l_{n}} \frac{d x}{s_{n}(x)^{3}}
$$

is the vessel resistance. The integration is along the vessel length $l_{n}$, measured by $x$, and $s_{n}(x)$ is the local cross-section. Lengths are normalized on the network's mother vessel cross-section $d^{*}$, so that $s_{1}=1$. We require $l_{n} \gg 1$, and now $R e=d^{*} U^{*} / \nu$, so that $l_{n} / R e$ is presumed to be $O(1)$ or more. The scale $U^{*}$ is implicitly defined through an insistence that $r_{1}=1$. The far-field result in (71) then stems from the Reynolds lubrication equation derived using $(4,13,21)$. In contrast, across a junction (the near-field) the pressure head $p+U^{2} / 2$ is constant, where $U$ is the averaged velocity. See (27-29). The pressure drop between the downstream ends of the parent, with subscript, $n$, and of a daughter, with subscript $m$ is

$$
\Pi_{m}=\frac{1}{2}\left(U_{m}^{2}-U_{n}^{2} / e_{n}^{2}\right)+r_{m} U_{m},
$$

where $e_{n}=\kappa_{n} / s_{n}$, which is greater than unity if a vessel widens along its length. The similar drop for the other daughter vessel, with subscript $l$, and mass conservation respectively give

$$
\Pi_{l}=\frac{1}{2}\left(U_{l}^{2}-U_{n}^{2} / e_{n}^{2}\right)+r_{l} U_{1}, \quad s_{n} U_{n}=s_{m} U_{m}+s_{l} U_{l} .
$$

These equations determine $U_{n}$, given a knowledge or guess of $\Pi_{m}, \Pi_{l}$. Knowing the total pressure drop across the network allows the simultaneous calculation of these local pressure drops and the velocities in each vessel. The resulting system is quadratically nonlinear and may be solved by Newton iteration.

Analytical progress can be made if the imposed pressure drops across the network are identical and the vessels at each generation $i$ are similar. The limit $N \rightarrow \infty$ with $N$ now the total number of generations and $i=q N, 0 \leq q<1,2 s_{i} / s_{i-1}=$ $(1-\tilde{b}(q) / N), r_{i}=\tilde{r}(q) / N, e_{i}=1+\tilde{e}(q) / N$, yields the ordinary differential equations

$$
d P / d q=\tilde{r} u+(\tilde{b}+\tilde{e}) u^{2}, d u / d q=\tilde{b} u,
$$

with $U_{i}=u(q)$ and with $P(q)$ denoting the pressure drop between the mouth and the current generation, as illustrated by (71-73). The change in subscript is to distinguish 


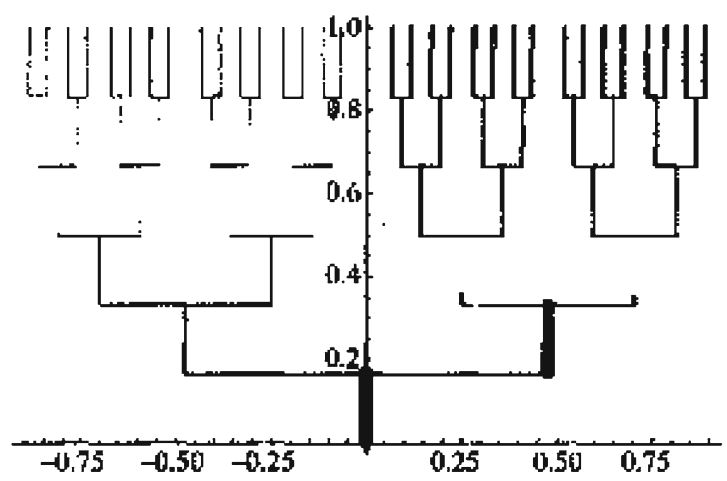

(i)
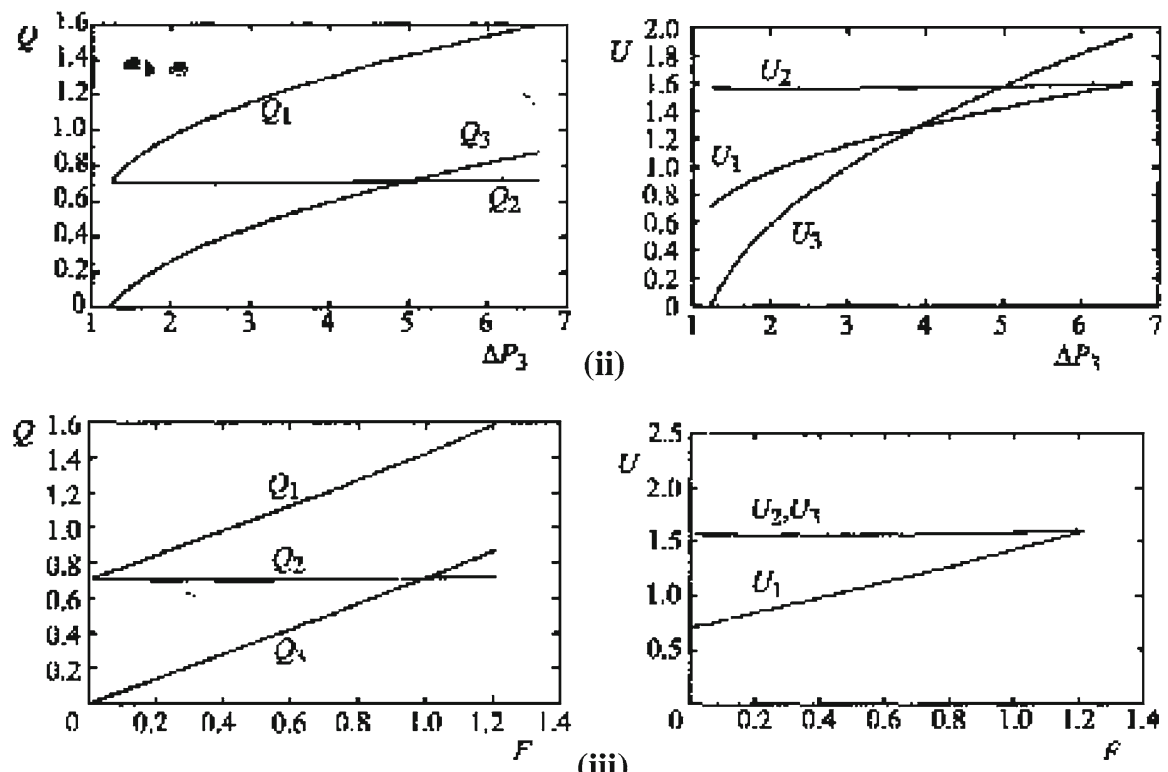

(iii)

Fig. 13 (i) (ii) (iii) Multi-generational branching model and analytical results

between generations rather than between vessels. The velocity $U_{0}$ into the parent is therefore related to the pressure drop $\Delta P$ across the network through the quadratic relation

$$
\Delta P=U_{0} \int_{0}^{1} \tilde{r} \tilde{\gamma} d q+U_{0}^{2} \int_{0}^{1}(\tilde{b}+\tilde{e}) \tilde{\gamma}^{2} d q, \quad \tilde{\gamma}(q)=\exp \left(\int_{0}^{q} \tilde{b}\left(q^{\prime}\right) d q^{\prime}\right)
$$

in this example. In less simple cases computation is necessary.

In two sample calculations based on (71-73) a single input vessel splits into 32 downstream (see Fig. 13(i)). In Fig. 13(ii) the viscous resistance $r$ for each vessel is 
half that at the previous generation, and at each bifurcation, the total cross-section of the network is reduced by a factor of 0.9 . We should recall that the individual vessel resistance depends on both width and length. Further, the pressure drop across the lefthand side of the tree is fixed at 5, while that across the right, $\Delta P_{3}$ is varied. The fluxes through each half, $Q_{2}, Q_{3}$, and through the whole network $Q_{1}$, are plotted together with the velocities into each vessel $U_{1,2,3}$. The minimum $\Delta P_{3}$ is approximately 1.25 , corresponding to no flow through the right side (zero flow at the first bifurcation). The upper value is approximately 6.65. Above this value, the flow decelerates into the left half and we can expect flow separation. In Fig. 13(iii) the pressure drop is 5 for all vessels, but in the right half each vessel has its cross-section increased by a factor $F$. The vessel lengths are varied so that the viscous resistance remains unaltered. The velocities at corresponding generations through the two halves of the network remain equal, although the fluxes do not. As $F \rightarrow 0$, the flux into the right branch vanishes as expected, although the velocity does not. For $F>1.22$, the flow decelerates into both halves at the first junction.

\subsection{Wall layers}

It is observed that viscous-inviscid interaction occurs in many of the wall layers present, notably those of Sects. 4, 5.1,6. The general features of wall layers are exemplified by those cases, in all of which the boundary-layer or wall-layer equations $(4,12,13)$ play an important part. The pressure remains unknown in advance or adjustable in each case. A related feature holds within the following section.

\section{Upstream influence}

It is enlightening to examine further the extent of upstream influence due to a branch junction in general, just as it is for a constriction as examined in Sect. 8 of [77], i.e. how far ahead is the incident flow substantially aware of the presence of a junction? The following sub-sections describe non-symmetric channel flow (Sect. 6.1), which admits a long range of upstream influence, developing networks (Sect. 6.2) and several other cases which allow only a shorter length of upstream effect (Sect. 6.3).

\subsection{In non-symmetric channels}

The case of viscous-inviscid interaction of [89] is on nonsymmetric branchings producing a network. This has the unusual axial scale

$$
|x| \sim R e^{1 / 7}
$$

mainly. The reason for the scale (75) originates from (35). In the core the velocity perturbation in $u$ is proportional to the displacement of $u_{0}(y)$ and so is of order $R e^{-1 / 3} x^{1 / 3}$, forcing a perturbation of order $R e^{-1 / 3} x^{-2 / 3}$ in $v$ by virtue of continuity. The $y$-momentum contribution then implies that $p \sim p_{y} \sim R e^{-1 / 3} x^{-5 / 3}$ since $y$ is 

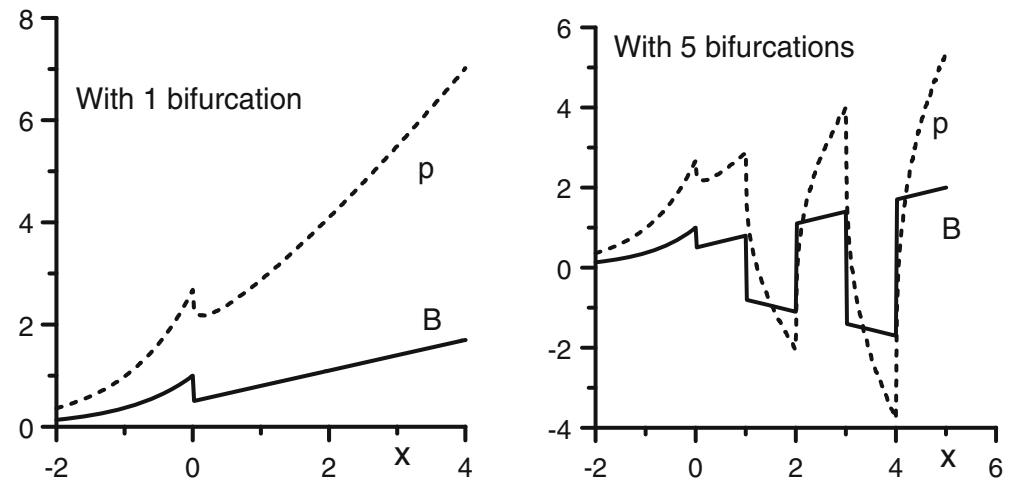

Fig. 14 Networks with upstream influence. Outer wall pressures $P$ and effective thicknesses $B$ for junctions at $x=0$ (left) and $x=0,1,2,3,4$ (right)

of $O(1)$. This pressure force should be compared with the induced near-wall pressure force which is of order $R e^{-2 / 3} x^{2 / 3}$ because of the inertia-pressure-viscous balance in the wall layer. The comparison gives us the result (75). The ensuing structure is all in one in a sense, giving a neat complete solution, but there is Euler-type jumping present as well, as follows.

Moving on to the issue of networks, then, we describe modelling of a planar network of bifurcating tubes as in Fig. 14 starting at $x=0$. The idea stems from constriction studies similar to those in Section 3. The upstream-influence length scale axially [43] is written $O\left(\epsilon^{-1}\right)$ where $\epsilon^{7}$ is $1 / R e$ and is small. The thin viscous layer at the lower outer wall near $y=0$ then has thickness of order $\epsilon^{2}$ and $(4,12,13)$ hold there in re-scaled terms with $R e$ replaced by unity. The boundary conditions required are

$$
\begin{aligned}
& u \sim \lambda_{0}(y+A(x)) \text { as } y \rightarrow \infty, \\
& \text { no slip at } y=f(x),
\end{aligned}
$$

where the positive constant $\lambda_{0}$ or $\lambda$ stands for the scaled incident wall shear and $f(x)$ now denotes the given lower-wall shape. If we ignore upstream influence for now (it is included subsequently), the negative boundary-layer displacement $A(x)$ can be obtained to within a factor related to the mass flux by the core-flow solution valid outside the wall layer; see below. In that case the viscous wall-layer problem determines the $\epsilon^{4}$ scaled wall pressure $p$ to within a constant.

\subsection{Developing analytical networks}

The article describes here 1-to-2 and various 1-to-many networks.

Suppose first that we have a single 1-to-2 branching. The inviscid core then within the lower daughter acts mostly as if distinct from that in the upper daughter and likewise for the viscous upper wall layer, over the present length scales. In the lower daughter core, the pressure is of order $\epsilon^{4}$ and the stream-function expands as 


$$
\Psi=\Psi_{0}(y)+\epsilon^{2}\left\{A(x) u_{0}(y)+\lambda_{2} \Psi_{0}(y)\right\}+\cdots
$$

where $\Psi_{0}$ is $\lambda_{0}\left(y^{2} / 2-y^{3} / 3\right)$ and $u_{0}$ is $\lambda_{0}\left(y-y^{2}\right)$, corresponding to the fully developed Poiseuille flow in the absence of any bifurcation, whereas the constant $\lambda_{2}$ is an unknown associated with the altered mass flux. The undeveloped viscous layers on the internal dividers of the daughters have negligible impact on the flow (they are passive as in Sect. 4.3), implying a tangential flow condition on the given divider underside $y=c_{0}-\epsilon^{2} T_{0}(x)$ say. Taking $T_{0}(0)$ as zero without loss of generality thus yields the classical thin-channel result

$$
A(x)=T_{0}(x)+K_{0}, \quad \text { for } x>0,
$$

(since $u_{0}\left(c_{0}\right)$ is nonzero) which determines the function $A(x)$ to within the additive constant $K_{0}$. Similarly, upstream influence present in the mother tube yields a freeinteraction behaviour [43]

$$
A(x)=K e^{\kappa x}, \quad \text { for } x<0,
$$

where $\kappa$ is a known positive constant and (3.4) represents an elliptic effect. (This problem of the 1-to- 2 case is treated by a Wiener-Hopf technique in $[44,85]$.) A novel feature due to the presence of the bifurcation (branching junction) however is that an axial jump in displacement can occur across the daughter entrances from $0-$ to $0+$, as in earlier examples. The jump is admissible and in fact necessary due to the set pressures upstream and downstream [61,90]. At the outer walls in particular, where the incident velocity is close to zero, the viscous layers allow the Bernoulli quantity $p+u^{2} / 2$ to be conserved as required along each local inviscid streamline as in Sect. 5.1 by means of a scaled pressure jump, in this case $\lambda_{0}^{2}\left(K^{2}-K_{0}^{2}\right) / 2$. The jumps are smoothed out over a shorter axial scale by an Euler region of length $O(1)$ in $x[61,90]$, which provides some direct communication between the two daughters and the mother. The feature that $K, K_{0}$ are unequal in general allows adjustment of $K_{0}$ in order to allow the lower-daughter pressure to satisfy the downstream pressure conditions, and likewise for the upper daughter.

Second, suppose a 1-to 4 network. Then another new feature appears as follows. Again attention can be restricted to a lower part, consisting now of a daughter described essentially earlier on and two granddaughters which begin at $x=x_{1}>0$. The lower of these granddaughters is also described essentially as before. The upper one however must suffer higher typical pressure variations of order $\epsilon^{2}$ such that

$$
\Psi=\Psi_{0}(y)+\epsilon^{2}\left\{D(x) u_{0}(y)+\lambda_{2}\left[\Psi_{0}(y)-\Psi_{0}\left(c_{1}\right)\right]\right\}+\cdots
$$

where $c_{1}-\epsilon^{2} T_{1}(x), c_{1}+\epsilon^{2} S_{1}(x)$ are the underside and topside respectively of the divider between these two granddaughters and

$$
D(x)=-p_{1}(x) \int u_{0}^{-2} d y-S_{1}(x)+\gamma_{1} .
$$


The integral is from $c_{1}$ to $y$, while the $\epsilon^{2}$ scaled pressure $p_{1}$ and the constant $\gamma_{1}$ are unknown. The novel feature here is that another jump must usually occur, namely in pressure across the entrance of the upper granddaughter from $x_{1}-$ to $x_{1}+$. This again is admissible, as the incident velocity is nonzero at all y heights of that granddaughter, allowing the Bernoulli property to be maintained along each streamline. This active jump is also smoothed out on a shorter axial scale by an $O$ (1) Euler region in $x-x_{1}$. (Overall this is another type of ellipticity.) As a result, it is found that a jump is also induced in the effective $A(x)$ function here which although still similar to $(79,80)$ now has

$$
A(x)=K e^{\kappa x}, \text { (discontinuity), } T_{0}(x)+K_{0}, \text { (discontinuity), } T_{1}(x)+K_{1}
$$

The doubly discontinuous form (83) then drives the viscous wall-layer response by means of the constraint (76). The displacement constants $K_{0}, K_{1}$ in (83) are controlled not only by the outermost (lower granddaughter) imposed pressure downstream but also by the inner (upper) granddaughter pressure imposed downstream.

Third, suppose a 1-to-8 network. Again consider its lower part. Yet another new feature enters as this new generation can contain some inner bifurcations which have nonzero incident velocity throughout and so can provoke the higher $O\left(\epsilon^{2}\right)$ pressure (and jumps) all the way across in $y$ as well as for long distances axially upstream and downstream, while outermost bifurcations continue the earlier established trend. One case, to focus attention, has the triply discontinuous form

$$
A(x)=\text { as in (83), then a discontinuity, then } T_{2}(x)+K_{2} \text {. }
$$

The three constants $K_{0}, K_{1}, K_{2}$ however depend on the four pressures imposed downstream in the four great-granddaughters (of this lower part) via the higher pressure responses and pressure jump occurring in the (implied) inner bifurcation as just described. The forms alternative to (84) in a 1-to-8 network depend on the relative positioning of each divider, making either (83) or a four-times discontinuous form hold.

Larger/generalized networks produce similar effects, i.e. potentially many discontinuities in the negative displacement $A(x)$ which, along with $f(x)$, forces the viscous layer by means of (76) and induces discontinuities in the wall pressure(s). The viscous layer is nonlinear in general, requiring numerical solution and admitting separation as in Sect. 3. By virtue of Prandtl's transposition theorem, the solution depends only on the effective thickness $(A+f)[=B$ say], thus giving wide application.

For small B, a linearized form applies and gives merely small discontinuities in pressure as in Fig. 14. A contraction of the outermost tube width broadly leads to a favourable pressure gradient and increasing wall shear, and expansion to an adverse pressure gradient with decreasing wall shear, as expected, but the discontinuities due to the branching junctions can counteract those trends. Concerning the biomedical applications mentioned in Sect. 1, the presence of multiple jumps in the solution(s) is likewise intriguing, especially if coupled with separation in nonlinear and/or unsteady cases. 


\subsection{Other cases}

With regard to other relevant cases, the recent work of [91] on two-phase flow through constrictions or branches is based on the ideas of the linear and nonlinear effects for the core and wall layers as studied in Sect. 4.3. The axial length scale here is that of order unity. Next, upstream influence is also seen in the exact branching-flow solution in Sect. 4.1, as well as in Sect. 4.3, both again with the axial scale being of order unity. Finally for now, we see upstream influence in the Euler-jump effects described in Sect. 4, whereas we have shorter length scales involved in the upstream influence of side branching, and in three dimensions the crucial length scales are broadly similar with only a few exceptions.

\section{In three dimensions}

Internal flows in three spatial dimensions are usually quite difficult to handle theoretically and the corresponding research area remains at the frontier in terms of understanding. For constriction there has been fair progress as in Sect. 7.1 below. For branchings part of the reason for the difficulty in theoretical progress is simply that in three dimensions fluid particles can move about so much more than in two dimensions. Thus within the inviscid setting of Sect. 4 for example we cannot readily say in advance whether a given fluid particle entering in the incident mother-vessel flow will end up inside daughter one or daughter two far downstream, in general. An exception may be made for particles moving (in the inviscid sense) along the walls. Nor can we say how much rotation there will be in the cross-sectional part of the motion in either of the daughters downstream. So analogues of the relations $(28,29)$ are not obtained readily.

We discuss some specific examples below.

\subsection{Constrictions}

We begin then with steady constricted flow. The first crucial size of three-dimensional constriction, in an otherwise straight pipe of circular cross-section $(r=1)$, is of height $O\left(R e^{-1 / 3}\right)$ in the radial $(r)$ direction with the length scales in the streamwise $(x)$ and azimuthal $(\theta)$ directions both being $O(1)$ : thus

$$
|x| \sim 1, \quad|\theta| \sim 1
$$

Then the three-dimensional boundary layer equations $(4,12,13)$ govern the viscous wall layer response, with the streamwise, radial and azimuthal velocities at leading order being

$$
R e^{-1 / 3} U,-R e^{-2 / 3} V, R e^{-1 / 3} W
$$

respectively, while $Y, Z$ stand for $R e^{1 / 3}(1-r), \theta$ in turn and the pressure is $R e^{-2 / 3} P$ $(x, \theta)+\cdots$. The no-slip condition applies to $U, V, W$ at the constriction surface $Y=h f(x, \theta)$ along with the outer condition 

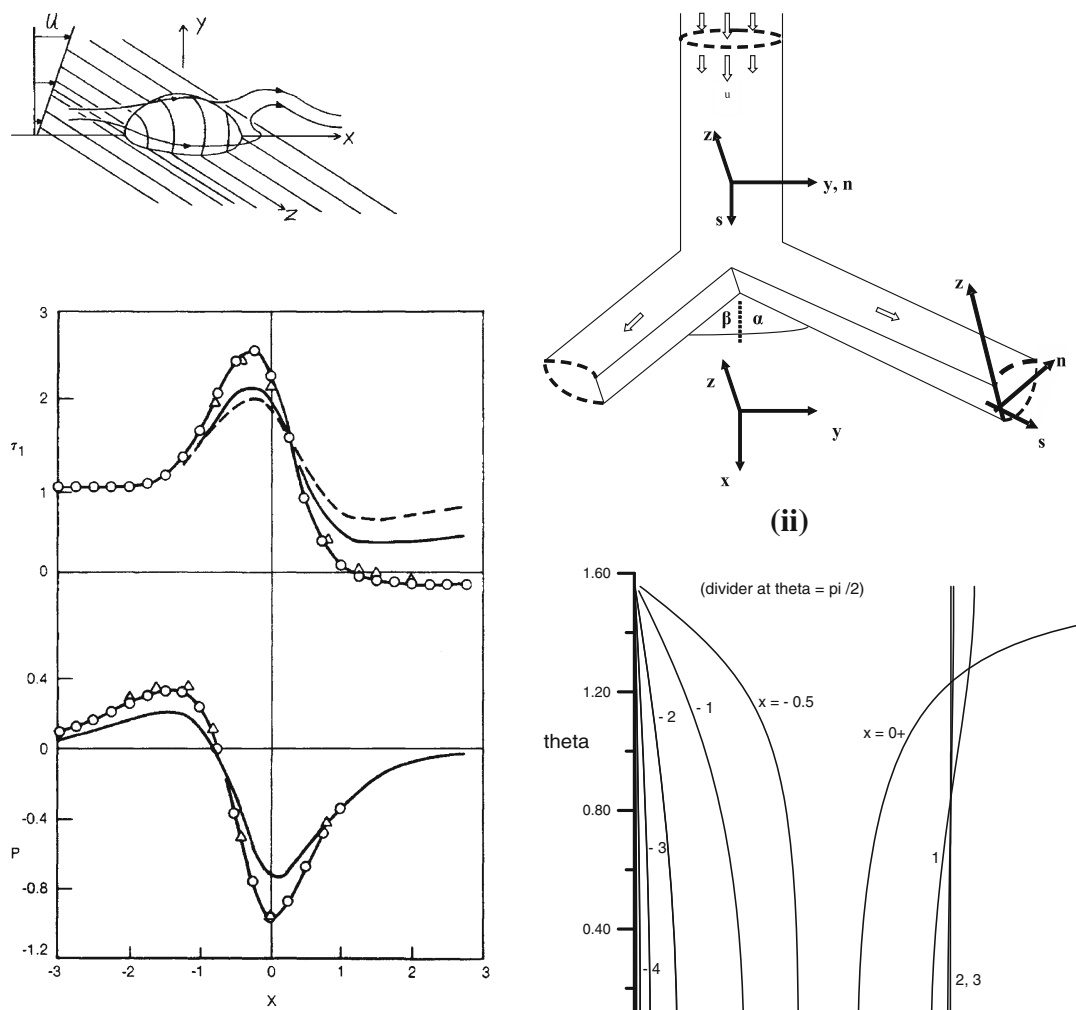

(i)

(ii)

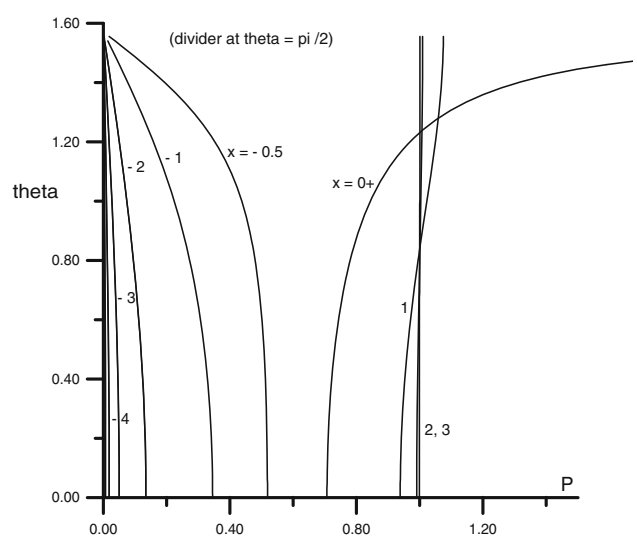

(iii)

Fig. 15 Three-dimensional flows. (i) For a constriction: typical particle trajectories; axial wall shear $\tau_{1}$ and pressure $P$ for height parameter $h=1$ (solid curves) and $h=1.5$ (circles, triangles, the latter to demonstrate grid effects); flow reversal is evident in the lee of the constriction for $h=1.5$ as the dashed curve then corresponds to $Z=\theta=1$ whereas all other curves are for $Z=\theta=0$. (ii) Diagram of bifurcation into two semi-circular daughters, as example of the situations in Sects. 7.2, 7.3: strictly the divergence angles are small but in practice the theory works for $\alpha, \beta$ up to $60^{\circ}$. (iii) For pressure-driven branching motion with junction at $x=0$ and two daughters: $\theta$-profiles of the induced wall pressure $P$ at different $x$ stations as indicated; the divider is situated at $($ thet $a=) \theta=\pi / 2$ and $-\pi / 2$ with solution symmetry about $\theta=0$

$$
U \sim Y+0, W \rightarrow 0 \text { as } Y \rightarrow \infty
$$

The lack of displacement for $U$ in (87) is necessary for consistency within the inviscid core flow where the oncoming motion, e.g. the Poiseuille form $u=\left(1-r^{2}\right) / 2$, is only slightly disturbed as in Sect. 3.1. The constriction surface is given by $r=$ $1-R e^{-1 / 3} h f(x, \theta)$ and the flow solution must have period $2 \pi$ in $\theta$.

As before, one of the primary aims is to keep track of the flow response as the disturbance increases in size. Thus [30] examines the linearized flow solutions holding for small $h$, followed by [28,29] (see also [92]) who calculate solutions for $h$ of $O(1)$ including some with regular three-dimensional separations present as in Fig. 15(i) 
and discuss the gradual change from three- to two-dimensional separated flow as the $\theta$-scale of the obstacle varies. The flow structure includes a non-linear upstream response which is three-dimensional in its origins since the corresponding two-dimensional flow of Sect. 3 produces no such upstream interaction. An investigation of this upstream response alone, appropriate to the next size of disturbance where $h$ is large, shows a singular termination occurring after a finite distance. The upstream flow becomes strongly attached in one part of the flow field after separating in another. This implies that the current flow structure is altered next by a feedback mechanism analogous to that of Sect. 3.2 for moderate constrictions. An extension of the reasoning there suggests that again, over the constriction, the pressure $P$ is generally large and $O\left(h^{2}\right)$ when $h \gg 1$, whereas upstream $P$ remains generally $O(1)$. The flow over the constriction here is dominated by the inviscid boundary layer forces, up to separation at least. So the next crucial stage is encountered when the constriction height rises to $O\left(R e^{-1 / 6}\right)$. It may be anticipated that the further adjustment required as the constriction height is increased beyond $O\left(R e^{-1 / 6}\right)$ is similar to that between Sects. 3.2 and 3.3 including the large upstream separation distance of (55).

\subsection{Branching over long scales}

The long scale of most interest for configurations such as that in Fig. 15(ii) has

$$
|x| \sim R e .
$$

The properties then are covered by $[63,93]$ and are based for steady three-dimensional flow on the asymptotic expansions

$$
[u, v, w, p]=\left[U, R e^{-1} V, R e^{-1} W, P(x)+R e^{-1} P_{1}\right]+\cdots,
$$

where $x=\operatorname{ReX} \sim \operatorname{Re}$ while $y, z$ are of $O(1)$, rather than on (86). These yield from $(4,5)$ at leading order the reduced nonlinear system

$$
\begin{aligned}
U U_{X}+V U_{y}+W U_{z} & =-P^{\prime}(x)+U_{y y}+U_{z z}, \\
U V_{X}+V V_{y}+W V_{z} & =-P_{1 y}+V_{y y}+V_{z z}, \\
U W_{X}+V W_{y}+W W_{z} & =-P_{1 z}+W_{y y}+W_{z z},
\end{aligned}
$$

along with the continuity equation (4). The system is that of a longitudinal vortex and, like many of the boundary-layer systems elsewhere in this article (a notable exception being the three-dimensional case in the previous section), it has the advantage of being parabolic in $X$ provided the velocity component $U$ remains positive. The working on it is mostly numerical in practice but useful local analysis is performed by $[63,93]$; see also $[62,94,95]$. In addition there is encouraging agreement between direct numerical solutions and the reduced system as shown in [63] for Re values in the low-to-mid 100s. 


\subsection{Branchings over short scales}

The short axial-length scales which are of most concern such as for Fig. 15(ii) are again given by

$$
|x| \sim 1
$$

but now in three dimensions. Short scales in general were addressed originally for constrictions as in Sect. 7.1, showing upstream influence; the present contribution is on current working [94] for three-dimensional slender branching and proceeds as follows.

Most previous theoretical or computational studies exclude the complexity associated with nonsymmetry in three-dimensional branching flows. There are notable exceptions to this among the many branching flow computations that have been performed, for instance among those concerned with predicting surgical graft properties, but they tend to be performed at a few isolated flow rates, divergence angles and so on, while on the theoretical side the only exceptions appear to be for low flow rates or for a side-branching at medium-to-high flow rates. Yet in reality and in experiments three-dimensional nonsymmetry is obviously the most common case of all the types of branching over a wide range of flow rates and other conditions. This is certainly so all the way through the human circulation system, including both the torso and the brain. The three-dimensional nonsymmetry of concern can be caused by unequal pressures at the downstream ends of the daughter vessels, by the branching geometry itself or by the incident velocity profiles in the mother being nonsymmetric. There are many other important specific influences such as the area ratios, divergence angles, wall roughness, wall flexibility, the number of daughter vessels and unsteadiness, although it is noted that flow pulsations are less significant in the brain for instance.

In fact little or no theoretical work on the subject of three-dimensional nonsymmetric branching flows at medium-to-high flow rates has been done as far as we know apart from $[21,63,95]$. The study [63] offers some promise based on the use of small-angle theory for a single mother tube of circular cross section dividing into two divergent daughters, where results are found to agree reasonably closely with direct simulations for angles of divergence up to $120^{\circ}$. Thus a surprisingly wide range of divergence angles was covered by the theory, over a range of medium flow rates: Reynolds numbers Re examined are in the low hundreds typically. Characteristic Re values of practical biomedical interest also include the 100s as mentioned in the introduction and divergence angles in reality are extremely varied.

The current investigation into three-dimensional nonsymmetric branching motions is concerned mainly with small disturbances and medium-to-high Reynolds numbers, based on [94]. This is partly because such disturbances seem the obvious first ones to address and partly because [63] suggests their usefulness could persist over a surprisingly broad range of flow conditions. In the investigation the end-pressures are assumed to apply over the relatively short axial length scale (93) and they are prescribed whereas in a fuller system they would be related to the long-scale pressure differences as remarked earlier. Shape effects and others are likewise considered over 
the same axial length scale. The one-to-two and one-to-many cases as before are both of major interest.

An effectively inviscid core is to be expected across the majority of the tube, along with a viscous (or more correctly a viscous-inviscid) layer at every wall present. Endpressure effects are found to act mostly at the outer wall of the branching configuration, whereas shape effects to consider are of two kinds, namely those due to the shape of the inner walls or dividers and those due to the outer wall shaping. Each kind of shape effect significantly impacts the wall layer behaviour also since the wall layer is especially sensitive, but there is a core effect too of course. So the core and the wall layer are examined in turn next.

In deriving the main scales and comparing the orders of magnitude of the dominant effects present, we may first take the small characteristic non-dimensional pressure magnitude $|p|$ as given, by virtue of the end-pressure constraints in the tubes. Expansions in small $R e^{-1}$ could be used as in Sect. 7.1 but here it is instructive to think in terms of $|p|$ instead. Then using arguments as in previous sections we concentrate on the range of flow rates for which

$$
1 \ll R e \ll|p|^{-3 / 2} .
$$

The reason for this is that, in intrinsic coordinates, a wall layer with small $|n|$ is expected, when $R e$ is large, in which the typical $u$ is $\lambda n$ plus a small disturbance $u_{1}$ say, where $u, \lambda n$ are equal to the first approximation because of the incoming wall shear $\lambda$. The orthogonal coordinates $\theta, n$ are tangential and inwardly normal to the outer walls respectively in the cross-plane. Similarly to Sect. 2.2 the main inertial, pressure-gradient and viscous forces are then represented in magnitude by $n u_{1},|p|, u_{1} /\left(\operatorname{Re} n^{2}\right)$ respectively, since $|x|$ is of $O(1)$. Hence $n$ must be of size $R e^{-1 / 3}$ typically, while $u_{1}$ is of size $R e^{1 / 3}|p|$ : this is smaller than the $\lambda n$ term as assumed provided that $R e$ lies within the range specified by (94). A numerical example is of immediate interest: if the non-dimensional pressure variation $|p|$ is as small as about 0.025 say then from (94) the range of $R e$ under consideration is from around 1 to 240, as a first estimate, a range which is certainly of some physical concern. Second, moving on to wall-shaping effects, the wall layer scalings above suggest examination of outer-wall shape distortions whose typical thickness is of the order $R e^{-1}|p|$, to compare directly against the influences of end-pressure. That in turn points to consideration of divider thicknesses of a similar size. The dividers however provoke pressures in the core flow which are larger than in the wall layer, because of the enhanced inertia in the core; compare section 4.3. Accordingly, if wall shaping is present, different sizes of pressure should be anticipated in the core and in the wall layer even when the shape distortions at the outer wall and at the dividers are comparable. The arguments just used serve to guide the solution expansions as follows, given that the mother pressure upstream scaled on $|p|$ is $P_{0}$ and the similarly scaled daughter pressures downstream are $P_{k}$, for integer $k$ running from 1 to $K$ say: there are thus $K$ daughters altogether and so $(K-1)$ dividers; we use $K$ instead of $N$ in this subsection because $N$ below denotes a scaled normal coordinate.

The core motion, being a perturbation of the incoming quasi-unidirectional motion with velocity profile denoted $u_{0}(y, z)$ and wall shear $\lambda(\theta)$ in the mother tube, has the expansion 


$$
[u, v, w, p]=\left[u_{0}(y, z), 0,0,0\right]+\epsilon_{1}\left[u^{\prime}, v^{\prime}, w^{\prime}, p^{\prime}\right](x, y, z, T)+\cdots
$$

where the coordinates $x, y, z, T$ are all of $O(1)$, the scaled velocity and pressure perturbations $u^{\prime}, v^{\prime}, w^{\prime}, p^{\prime}$ are also of $O(1)$, and $\epsilon_{1}$ is small. The amplitude factor $\epsilon_{1}$ is in fact usually larger than $|p|$ above (in view of the different pressure scales described in the previous paragraph) and must also be greater than or equal to $R e^{-1 / 2}$ nominally because of the Blasius-like viscous thickness of Sect. 2.2 that is generated on each divider inside the core flow. The time scale $t_{s}$ is assumed to be large; later we specify it precisely. Hence from substitution into the Navier-Stokes equations of Sect. 2.1 the flow response is quasi-steady here, giving the governing equations for the vector $\underline{u}^{\prime}$ defined by $\underline{u}^{\prime}=\left(u^{\prime}, v^{\prime}, w^{\prime}\right)$ as

$$
\begin{gathered}
\underline{\nabla} \cdot \underline{u}^{\prime}=0, \\
u_{0} \underline{u}_{x}^{\prime}+\left(v^{\prime} u_{0 y}+w^{\prime} u_{0 z}, 0,0\right)=-\underline{\nabla} p^{\prime},
\end{gathered}
$$

from the mass conservation and momentum balances. Here $\underline{\nabla}$ stands for the gradient operator $\left(\partial_{x}, \partial_{y}, \partial_{z}\right)$. A faster time scale of order unity would add an acceleration term $\underline{u}_{t}^{\prime}$ to the left-hand side of $(97)$. It follows from $(96,97)$ that the equation

$$
\nabla^{2} p^{\prime}=2\left(u_{0 y} p_{y}^{\prime}+u_{0 z} p_{z}^{\prime}\right) / u_{0}
$$

holds for the pressure perturbation $p^{\prime}$ (compare the two-dimensional version of Sects. $3.1,4.3)$ subject to the conditions

$$
\begin{gathered}
p^{\prime} \rightarrow 0 \text { as } x \rightarrow \infty \text { or }-\infty, \\
\partial p^{\prime} / \partial n=-u_{0}^{2} \partial^{2} f_{k}^{+} / \partial x^{2} \text { at } y=y_{k}+, \text { and }-u_{0}^{2} \partial^{2} f_{k}^{-} / \partial x^{2} \text { at } y=y_{k}^{-}, \\
p^{\prime} \rightarrow 0 \text { as } n \rightarrow 0 \text { at outer wall. }
\end{gathered}
$$

These boundary conditions are analogous to those in the earlier studied two-dimensional scenario and reflect respectively the upstream and downstream end pressures (the zeros seen upstream and downstream in (99) echo the earlier discussion on different pressures), the tangential flow conditions on the dividers, and the necessary matching with the wall layer flow. The $f_{k}$ values represent the scaled thicknesses of the $K-1$ internal dividers and $y_{k}$ their locations: these are the main shaping effects within the core. Thus the size of $\epsilon_{1}$ allows for the first appearance of shape effects, in (100), but its relative largeness accounts for the condition (99). The condition (100) in detail stems from the requirement of tangential flow via the momentum balances, while the wall-layer matching condition (101) is considered in more detail below. Local analysis shows that $p^{\prime}$ behaves as $\lambda\left(\partial^{2} A / \partial x^{2}\right) n^{3} / 3$ near $n$ zero, where the function $A(x, \theta, T)$ represents the shaping effects from the core. In the approach to the outer wall, that all leaves $v_{1}^{\prime} \propto n, p^{\prime} \propto n^{3}, w_{1}^{\prime} \propto n^{2}$ but a tangential slip velocity

$$
u^{\prime} \rightarrow A(x, \theta, T) \text { as } n \rightarrow 0
$$


in the axial direction. Here $v_{1}=\epsilon_{1} v_{1}^{\prime}, w_{1}=\epsilon_{1} w_{1}^{\prime}$ to leading order are the velocity components in the $n, s$ directions in turn. We note in passing that a logarithmic accumulation which can occur at any junction of outer wall and divider is of little import here since the perturbations in general are small.

In the viscous wall layer the implied flow solution is expressed in the form

$$
\begin{aligned}
& {\left[u, v_{1}, w_{1}, p\right]} \\
& \quad=\left[R e^{-1 / 3} \lambda N, 0,0,0\right]+\epsilon_{2}\left[R e^{-1 / 3} U, R e^{-2 / 3} V_{1}, R e^{-1 / 3} W_{1}, R e^{-2 / 3} P\right]+\cdots
\end{aligned}
$$

where $n=R e^{-1 / 3} N$. Here for now we have the parameter $\epsilon_{2}$ just being arbitrarily small. The outer wall itself is prescribed by $n=\epsilon_{2} R e^{-1 / 3} F(x, z, T)$, giving $N=\epsilon_{2} F(x, z, T)$. The orders of the velocities and pressure in (103) are required for conservation of mass and momentum. The time scale specifically has $t=R e^{1 / 3} T$ (so $t_{s}$ is identified with $\mathrm{Re}^{1 / 3}$ ) to affect the wall layer dynamics and is thus relatively slow as supposed in the argument above, whereas $x, \theta$ are again of order unity.

Hence, upon substitution into the Navier-Stokes equations, the unsteady viscous three-dimensional wall-layer equations are found to apply,

$$
\begin{aligned}
& U_{x}+V_{1 N}+W_{1 \theta}=0, \\
& U_{T}+\lambda N U_{x}+V_{1} \lambda+W_{1} \lambda_{\theta} N=-P_{x}+U_{N N}, \\
& W_{1 T}+\lambda N W_{1 x}=-P_{\theta}+W_{1 N N},
\end{aligned}
$$

with the unknown scaled pressure $P(x, \theta, T)$ being independent of $N$ because the normal momentum balance requires $\partial P / \partial N$ to be zero at leading order. The system above is as in $(4,12,13)$ but linearised. The boundary conditions on the wall-layer system are

$$
\begin{aligned}
& U=-\lambda F, V_{1}=W_{1}=0 \text { at } N=0, \\
& U \rightarrow A(x, \theta, T), W_{1} \rightarrow 0 \text { as } N \rightarrow \infty, \\
& \left(U, V_{1}, W_{1}\right) \rightarrow 0, P \rightarrow P_{0}(T) \text { as } x \rightarrow-\infty, \\
& P \rightarrow P_{k}(T) \text { as } x \rightarrow \infty \text { in the } k \text { th daughter. }
\end{aligned}
$$

The scaled mother pressure $P_{0}(T)$ and the scaled daughter pressures $P_{k}(T)$ are those that were introduced earlier on, although now with explicit allowance for their possible unsteadiness. Also, from matching, $\epsilon_{2} R e^{-1 / 3}$ is identical with $|p|$ and hence with $\epsilon_{1}$. The boundary conditions above reflect the no-slip conditions at the distorted wall (along with a Taylor expansion bringing in the scaled outer wall shaping $F$ ), in (107), the match to the core in (108) (this leads to an algebraic decay in $Y$ ), and the upstream and downstream pressure conditions. Shaping effects are present in the contributions $A, F$, due to the core and the outer wall in turn. To repeat, we have taken the pressure and shape to have comparable effects in the wall layer of relative amplitude $O\left(\epsilon_{2}\right)$ at this stage. Further, a mild assumption that $\epsilon_{2}$ is much larger than $R e^{-1 / 3}$ has been made; otherwise an extra contribution $\frac{1}{2} \mu N^{2} R e^{-2 / 3}$ is needed in the expression for 
$u$ in (103) to account for the incident $O(1)$ velocity profile curvature $\mu$ and associated with that an extra term $-\mu R e^{-1} x+$ constant in the pressure $p$. Implicit also throughout all this is the requirement of

$$
\text { periodicity in } \theta
$$

for all the velocities and pressures, since $\theta$ is in the azimuthal direction.

Several points need to be made at this stage. First the shape effects as seen in the wall layer are clearly two-fold, coming not only from $A(x, \theta, T)$ which represents the influence of the divider shaping in the core but also from $F(x, \theta, T)$ due directly to the outer wall shape. Again, the slip velocity $A$ acts in the wall layer as a given negative displacement, in view of the contributions to the axial velocity in (103) and the condition (108). Shape effects will be mentioned in Sect. 8. The end-pressure (or pressure-driven) effects are in contrast to the shape ones in the sense that the former are confined to the wall layer to leading order. Such pressure-driven flows have $A$ being zero and so are un-influenced directly by any pressure $p^{\prime}$ in the core. Instead the induced wall pressure in such cases dictates what the core pressure response $p^{\prime}$ must be, allowing for an amplitude factor reduced from that in the general case (95). Finally here, the argument also extends to unsteady motions and likewise allows for non-uniform $\lambda(\theta)$ as above if necessary, as well as other realistic effects.

\section{Pressure-driven flows}

The configuration for pressure-driven flows applies for two or more daughters where the flow is driven merely by pressure differences, so that the shape-effect forcings from the core and the outer wall are all absent. Thus $F$ and $A$ in $(107,108)$ are both zero here. Steady flow is assumed for now. The wall-layer flow problem set out above has an unusual solution then in that $V_{1}$ is identically zero, and hence adding the $x$ derivative of (105) to the $\theta$ derivative of (106) yields Laplace's equation

$$
\nabla_{2}^{2} P=0
$$

for the unknown outer-wall pressure $P(x, \theta)$, where $\nabla_{2}^{2}$ denotes the two-dimensional Laplacian $\partial_{x}^{2}+\partial_{\theta}^{2}$. The boundary conditions to be satisfied by $P$ are those of the prescribed pressures far upstream and downstream as written in $(109,110)$, supplemented by the requirement (111) at all finite $x$ stations. The reduction of the originally three-dimensional wall-layer flow problem to the two-dimensional Laplacian problem (112) is notable.

Being driven by spatially constant end-pressures acting up- and downstream means that the pressure response in between involves eigensolutions in effect. These can be seen in the following basic case of two daughters, $K=2$, with end pressures $P_{1}, P_{2}$ in the daughters such that $P_{2}=-P_{1}$. Here there is an exact solution which stems from a conformal mapping, such that

$$
P / P_{1}= \pm \operatorname{real}\left(1+e^{-2 \varsigma}\right)^{-1 / 2}
$$


where $\varsigma$ denotes $x+i \theta$ and the branching starts at zero $x$ with the junctions of the outer walls and the divider being symmetrically disposed at $\theta$ values equal to $\pi / 2$ and $3 \pi / 2$. This is similar to the finding in Sect. 4.1. The solution exhibits the irregular response of the pressure near the leading edge of the divider in the present threedimensional nonsymmetric-flow setting, a response which is more severe than in the symmetric-flow setting of [59] despite the geometric shapes studied being identical. There is also the series representation

$$
\begin{aligned}
& P / P_{1}=e^{x} \cos \theta-(1 / 2) e^{3 x} \cos 3 \theta+(3 / 8) e^{5 x} \cos 5 \theta+\cdots \text { for } x<0, \\
& P / P_{1}=1-(1 / 2) e^{-2 x} \cos 2 \theta+(3 / 8) e^{-4 x} \cos 4 \theta+\cdots \text { for } x>0,
\end{aligned}
$$

which shows the pressure eigenforms clearly. Here (115) applies in the daughter with end-pressure $P_{1}$, whereas the pressure in the other daughter is equal and opposite. The wall pressure responses are presented in Fig. 15(iii).

For more than two daughters, $K>2$, conformal mapping or series solutions can again be applied. The wall pressure solutions $P$ are given by [94] for a range of values of $K$, along with velocity profiles and the induced scaled wall-shear distributions obtained analytically from (104-106), with $\lambda$ constant, $T$ dependence being absent and with $P$ found as just above. For instance the integral form

$$
\begin{aligned}
\tau_{1}= & \phi \int_{-\infty}^{x}(x-\xi)^{-2 / 3} \frac{\partial P}{\partial \xi}(\xi, \theta) d \xi, \\
& \text { with } \lambda^{1 / 3} \phi=-3^{1 / 6}(\Gamma(2 / 3))^{2} /(2 \pi)=-0.35047 \ldots,
\end{aligned}
$$

determines the axial shear stress perturbation $\tau_{1}$ and a similar integral yields the cross-plane shear stress $\tau_{2}$, where $\tau_{1}, \tau_{2}$ are the values of $\partial U / \partial N, \partial W_{1} / \partial N$ at the wall $N=0$, respectively.

The main effects present in the wall-layer results are those of different effective suctions into the daughter tubes as they compete with each other to draw fluid from the mother tube. In every case a sufficiently low end-pressure downstream in a daughter tends to draw fluid into that daughter, causing an increase in the corresponding axial shear stress and directions of the neighbouring cross-plane shear stresses that indicate cross-plane movement of fluid towards the lower-pressure daughter. The maximal effects in the wall shear stresses occur quite close to the daughter entrances. Upstream of the entrances the wall shear stress response is essentially exponential in form whereas downstream the response involves a comparatively slow algebraic $\left(x^{-1 / 3}\right)$ decay, consistent with (116). The dominant response sufficiently far upstream is seen to be an axially symmetric one, by the way. Extremes can also be investigated as in [59] such as for large values of $K$, where the wall pressure problem becomes approximately one holding in negative $x$ alone, and for small daughter-widths which lead to a global sink influence accompanied by a local region close to the entrance of the daughter tube.

The flow configuration so far has the sum of the end pressures being equal to zero. If the sum is nonzero, then consideration can be given to adding in an extra unknown 
mass flux, corresponding to a small contribution proportional to $n^{2}$ in $u_{0}$ near the wall. Varying gap-widths may also be incorporated as suggested earlier. More significantly, it is possible to move on to find the core solution forced by the known wall pressures determined as above and hence predict the swirl provoked in the core due to the pressure-driven configuration. Here the amplitude of the velocity and pressure perturbations is of typical size $\epsilon_{2} R e^{-2 / 3}$, from (103), rather than the size $\epsilon_{1}$ in (95), and so the newly scaled core pressure $p^{\prime}$ satisfies (98) but subject to generally nonzero downstream end pressures instead of (99), and zero normal pressure gradient conditions replace (100) at the dividers, while (101) is replaced by

$$
p^{\prime} \rightarrow P(x, s) \text { as } n \rightarrow 0 \text { at the outer wall. }
$$

Given (117), the tangential components in the velocity perturbations grow like $1 / n$ as the outer wall is approached. The corresponding core solutions are shown in [94].

\section{Further comments}

The internal-flow research described herein has been almost entirely on (a) constrictions, (b) branchings as discussed at the very start of the article. The aim has been to seek out relatively simple configurations and basic properties first, given that the occurrence of important nonlinear effects implies mathematical accounts are often likely to be insightful. Assumptions made for convenience or for making headway are mentioned earlier and include those of mostly steady laminar incompressible-fluid motions over medium-to-large ranges of the Reynolds number. We believe the study may be of interest in terms of mathematical issues, real applications, the science of fluid dynamics and the clear interaction with direct numerical simulations.

In reality there are often very complex networks to deal with in the practical situations of concern. Reconnections in addition to branchings are of interest whether predominantly inviscid or viscous-inviscid in nature. Flexible walls also arise in reality and, among other possibilities, they lead to integral equations stemming from (63) for instance. Again, the regime of small Reynolds numbers also has much fascination. The current contribution is further related to continuing work on many-body problems motivated by industrial as well as biomedical applications.

There are many other follow-on studies which could be undertaken. With that in mind we finish with a number of suggestions, issues and questions, small or large, which we believe are of wide interest.

8.1 Explore the exact solution for the 1-to-2 branching described in Sect. 2.2. What happens physically if there is no such solution?

8.2 Explore the 1-to-4 case of Sects. 2.2, 4.2. Does it give the same as 1-to-2 followed by 2 -to-4, i.e. 1 mother to 2 daughters to 4 grand-daughters?

8.3 Investigate the case of 1 to $N$ branching for general values of $N$ in Sect. 4.2. Deduce what occurs in the limit of large $N$. 
8.4 For fluid motion through a constricted tube (Sects. 3, 6), describe the flow structure and solution in the presence of an incident velocity profile which is not symmetric in the lateral direction. That is in two dimensions.

8.5 For three-dimensional fluid motion through a constricted tube (Sect. 7.1), write down the flow structure and solution in the presence of an incident velocity profile that is not symmetric in the generalised azimuthal direction.

8.6 Explore the asymptotic behaviour of the $\bar{A}$-formula in Sect. 4.4 for large $N$.

8.7 Derive the equation balancing $u_{0} \nabla^{2} \psi$ and $u_{0}^{\prime \prime} \psi$ in the core of Sect. 3.1.

8.8 Go through the analysis of the core flow of Sect. 3.1 in detail.

8.9 Examine the solution features for large values of $N$ in Sect. 5.2, concerning ever larger networks.

8.10 Investigate the solution for non-symmetric constriction analogous to that in Sect. 8 of [77]. Can this be extended to wall-injection of fluid? Can it also be extended to branching flows?

8.11 Study upstream influence in channel flows which lack symmetry, based on an appropriate balance between the scaled wall pressure difference $p_{\text {upper }}-p_{\text {lower }}$ and the scaled core displacement curvature $A^{\prime \prime}$.

8.12 Seek linearised analytical solutions for the otherwise nonlinear problems described in Sects. 3.1-3.3, 5.1, 6.1, concerning two-dimensional motions.

8.13 Derive the main equation of Sect. 4.4 for the area $\bar{A}$.

8.14 Go through the details of Sect. 4.4.

8.15 Explore the $A+F, A-G$ effects due to relative displacements that come into the reckoning for length scales $|x|$ exceeding the $1 / 7$ scale of (80).

8.16 Investigate the case [27] corresponding to severe constriction of a non-symmetric channel. Apply this to branching situations if possible.

8.17 Compute solutions for the nonlinear problems described above.

8.18 Study further the unsteady flows considered in [26]. Unsteady flows are especially interesting with pulsatile incident motions in a mother vessel for instance and analysis can be applied for many of the configurations addressed in this article.

8.19 Examine the unsteady version of the triple deck problem and its significance in terms of linear and nonlinear Tollmien-Schlichting waves and breakdown [96].

8.20 Examine the unsteady version of the nonsymmetric channel flow problem (Sect. 6) and its significance in terms of linear and nonlinear viscous-inviscid waves and breakdown [96].

8.21 Investigate the effects of entry flow [44] on constricted or branched vessels.

8.22 Address the splitter-plate study of [58].

8.23 Consider re-connections of branched vessels, such as in a scenario with 1-to- $n$ branching followed downstream by an $n$-to- 1 re-connection.

8.24 For constricted three-dimensional motions as in Sect. 7.1 perform a linearised analysis. Show that there is an upstream response ahead of the constriction.

8.25 Does the axi-symmetric component dominate the highly nonlinear response upstream of a severe constriction even in the three-dimensional situation of Sect. 7.1? 
8.26 Extend the analysis of three-dimensional branching motions in Sect. 7.3 to allow for the end pressures being dependent on the scaled time. The major effects occur in the scaled wall shears.

8.27 Analyse the effect of an incident velocity profile that is not symmetric in the generalised azimuthal direction, for three-dimensional branching motion as in Sect. 7.3.

8.28 Examine and compare the influences of geometrical shape (divergence angles, thickness, camber, positioning) in two- and three-dimensional branchings.

Acknowledgments Thanks are due to many colleagues for their interest or collaboration in parts of this article. Thanks also go to a referee and the editor for helpful comments and to journals for kind permission to show certain figures.

Open Access This article is distributed under the terms of the Creative Commons Attribution License which permits any use, distribution, and reproduction in any medium, provided the original author(s) and the source are credited.

\section{References}

1. Pedley, T.J.: Mathematical modelling of arterial fluid dynamics. J. Eng. Math. 47, 419-444 (2003)

2. Grotberg, J.B., Jenson, O.E.: Biofluid mechanics in flexible tubes. Annu. Rev. Fluid Mech. 36, 121-147 (2004)

3. van de Vosse, F.N.: Mathematical modelling of the cardiovascular system. J. Eng. Math. 47, 175-183 (2003)

4. Mulder, G., Bogaerds, A.C.B., Rongen, P., van de Vosse, F.N.: On automated analysis of flow patterns in cerebral aneurysms based on vortex identification. J. Eng. Math. 64(4), 391 (2009)

5. Pedley, T.J.: The Fluid Mechanics of Large Blood Vessels. Cambridge University Press, Cambridge (1980)

6. Wootton, D.M., Ku, D.N.: Fluid mechanics of vascular systems, diseases, and thrombosis. Ann. Rev. Eng. 1, 299-329 (1999)

7. Alastruey, J., Parker, K.H., Peiró, J., Sherwin, S.J.: Can the modified Allen's test always detect sufficient collateral flow in the hand? A computational study. Comput. Methods Biomech. Biomed. Eng. 9, 353-361 (2006)

8. Griffiths, D.J.: Urodynamics—The Mechanics and Hydrodynamics of the Lower Urinary Tract. Hilger, Bristol (1980)

9. Xu, Q., Rossen, W.R.: Effective viscosity of foam in periodically constricted tubes. Colloids Surf. A: Physicochem. Eng. Asp. 216(1-3), 175-194 (2003)

10. Itoh, H., Smaldone, G.C., Swift, D.L., Wagner, H.N. Jr.: Quantitative evaluation of aerosol deposition in constricted tubes. J. Aerosol Science 16(2), 167-174 (1985)

11. Rossen, W.R.: Snap-off in constricted tubes and porous media. Colloids Surf. A: Physicochem. Eng. Asp. 166(1-3), 101-107 (2000)

12. Zhang, P., Hinshaw, J.E.: Three-dimensional reconstruction of dynamin in the constricted state. Nat. Cell Biol. 3, 922-926 (2001). http://cellbio.nature.com

13. Ding, Y.J., Lieber, B., Yu, C.P.: Motion of a fiber in a bifurcating tube flow. Aerosol Sci. Technol. 18(1), 100-103 (1993). doi:10.1080/02786829308959587

14. Shi, H., Kleinstreuer, C., Zhang, Z., Kim, C.S.: Nanoparticle transport and deposition in bifurcating tubes with different inlet conditions. Phys. Fluids 16, 2199 (2004)

15. McEvoy, A.W., Kitchen, N.D., Thomas, D.G.T.: Intracerebral haemorrhage in young adults: the emergence of drug misuse. BMJ 320, 1322-1324 (2000)

16. Olufsen, M.S., Peskin, C.S., Kim, W.Y., Pedersen, E.M., Nadim, A., Larsen, J.: Numerical simulation and experimental validation of blood flow in arteries with structured-tree outflow conditions. Ann. Biomed. Eng. 28, 1281-1299 (2000)

17. Quarteroni, A., Formaggia, L., Veneziani, A. (eds.): Cardiovascular Mathematics. Springer, Berlin. http://www.springer.com/math/applications/book/978-88-470-1151-9 (2009) 
18. van de Vosse, F.N., de Hart, J., van Oijen, C.H.G.A., Bessems, D., Segal, A., Wolters, B.J.B.M., Stijnen, J.M.A., Baaijens, F.P.T.: Finite-element-based computational methods for cardiovascular fluid-structure interaction. J. Eng. Math. 47, 335-368 (2003)

19. Caro, C.G., Doorly, D.J., Tarnawski, M., Scott, K.T., Loy, Q., Dumoulin, C.L.: Non-planar curvature and branching of arteries and non-planar type flow. Proc. Roy. Soc. A 452, 185-197 (1996)

20. Resnick, N., Einav, S., Chen-Konak, L., Zilberman, M., Yahav, H., Shay-Salit, A.: Haemodynamic forces as a stimulus for arteriogenesis. Endothelium 10, 197-206 (2004)

21. Blyth, M.G., Mestel, A.J.: Steady flow in a dividing pipe. J. Fluid Mech. 401, 339-364 (1999)

22. Lighthill, M.J.: Physiological fluid dynamics: a survey. J. Fluid Mech. 52, 475-597 (1972)

23. Alastruey, J., Moore, S.M., Parker, K.H., David, T., Peiró, J., Sherwin, S.J.: Reduced modelling of blood flow in the cerebral circulation: coupling 1-D, 0-D and cerebral auto-regulation models. Int. J. Numer. Methods Fluids 56, 1061-1067 (2008)

24. Passerini, T., de Luca, M., Formaggia, L., Quarteroni, A., Veneziani, A.: A 3D/1D geometrical multiscale model of cerebral vasculature. J. Eng. Math. 64(4), 391 (2009)

25. David, T., Alzaidi, S., Farr, H.: Coupled autoregulation models in the cerebro-vasculature. J. Eng. Math. 64(4), 403 (2009)

26. Smith, F.T.: Flow through constricted or dilated pipes and channels. Part 1. Q. J. Mech. Appl. Math. XXIX, Pt. 3 (1976)

27. Smith, F.T., Duck, P.W.: On the severe nonsymmetric constriction, curving or cornering of channel flows. J. Fluid Mech. 90(4), 727-753 (1980)

28. Sykes, R.I.: On three-dimensional boundary layer flow over surface irregularities. Proc. R. Soc. A 373, 311-329 (1980)

29. Smith, F.T.: Properties, and a finite difference approach, for interactive three dimensional boundary layers. UTRC 83-46 (1983) [see also Steady and unsteady boundary layer separation. Ann. Rev. Fluid Mech. 18, 197-220 (1986)]

30. Smith, F.T.: Pipeflows distorted by non symmetric indentation or branching. Mathematika 23, 62-83 (1976)

31. Augst, A.D., Ariff, B., McG Thom, S.A.G., Xu, X.Y., Hughes, A.D.: Analysis of complex flow and the relationship between blood pressure, wall shear stress, and intima-media thickness in the human carotid artery. Am. J. Physiol. Heart Circ. Physiol. 293(2), H1031-H1037 (2007)

32. Augst, A.D., Barratt, D.C., Hughes, A.D., Glor, F.P., McG Thom, S.A.G., Xu, X.Y.: Accuracy and reproducibility of CFD predicted wall shear stress using 3D ultrasound images. J. Biomech. Eng. 125(2), 218-222 (2003)

33. Gao, E., Young, W.L., Pile-Spellman, J., Joshi, S., Duong, H., Stieg, P.E., Ma, Q.: Cerebral arteriovenous malformation feeding artery aneurysms; a theoretical model of intravascular pressure changes after treatment. Neurosurgery 41, 1345-1358 (1997)

34. Hademenos, G.J., Massoud, T.F.: Biophysical mechanisms of stroke. Stroke 28, 2067-2077 (1997)

35. Brada, M., Kitchen, N.D.: How effective is radiosurgery for arteriovenous malformations? J. Neurol. Neurosurg. Psychiat. 68, 548-549 (2000)

36. Handa, T., Negero, M., Miyachi, S., Sugita, K.: Evaluation of pressure changes in feeding arteries during embolization of intracerebral arteriovenous malformations. J. Neurosurg. 79, 383-389 (1993)

37. Miyasaka, Y., Yada, K., Kurata, A., Tokiwa, K., Irikura, K., Tanaka, R., Ohwada, T., Kitahara, T.: Correlation between intravascular pressure and risk of haemorrhage due to arteriovenous malformations. Surg. Neurol. 39, 370-373 (1993)

38. Young, W.L., Kader, A., Pile-Spellman, J., Ornstein, E., Stein, B.M.: Arteriovenous malformation drawing vein physiology and determinants of transnidal pressure gradients. Neurosurgery $\mathbf{3 5}$, 389-396 (1996)

39. Cebral, J.R., Putman, C.M., Alley, M.T., Hope, T., Bammer, R., Calamante, F.: Hemodynamics in normal cerebral arteries: qualitative comparison of 4D phase-contrast magnetic resonance and imagebased computational fluid dynamics. J. Eng. Math. 64(4), 367 (2009)

40. Pranevicius, O., Pranevicius, M., Liebeskind, D.S.: Partial aortic occlusion and cerebral venous steal. Stroke 42, 1478-1481 (2011)

41. Lasheras, J.C.: The biomechanics of arterial aneurysms. Ann. Rev. Fluid Mech. 39, 293-319 (2007)

42. Smith, F.T.: Flow through constricted or dilated pipes and channels. Part 2. Q. J. Mech. Appl. Math. XXIX, Pt. 3 (1976)

43. Smith, F.T.: Upstream interactions in channel flows. J. Fluid Mech. 79(4), 631-655 (1977) 
44. Smith, F.T.: On entry flow effects in bifurcating, blocked or constricted tubes. J. Fluid Mech. 78(4), 709-736 (1976)

45. Pedley, T.J., Luo, X.Y.: Modelling flow and oscillations in collapsible tubes. Theor. Comput. Fluid Dyn. 10, 277-294 (1998)

46. Plotkin, A., Mei, R.W.: Navier-Stokes solutions for some laminar incompressible flow with separation in forward facing step geometries. AIAA J. 24(7), 1106-1111 (1986)

47. Sobey, I.J.: On flow through furrowed channels. J. Fluid Mech. 96, 1-26 (1980)

48. Pedley, T.J., Corieri, P., Kamm, R.D., Grotberg, J.B., Hydon, P.E., Schroter, R.C.: Gas flow and mixing in the airways. Crit. Care Med. 22, 24-36 (1994)

49. Smith, F.T., Green, J.E.F., Ovenden, N.C.: Flow in a multi-branching vessel with compliant walls. J. Eng. Math. 64(4), 353-365 (2009)

50. Smith, F.T., Purvis, R., Dennis, S.C.R., Jones, M.A., Ovenden, N.C., Tadjfar, M.: Fluid flow through various branching tubes. J. Eng. Math. 47, 277-298 (2003)

51. Wilquem, F., Degrez, G.: Numerical modelling of steady inspiratory airflow through a three-generation model of the human central airways. J. Biomech. Eng. 119, 59-65 (1997)

52. Goldman, D., Popel, A.S.: A computational study of the effect of capillary network anastomoses and tortuosity on oxygen transport. J. Theor. Biol. 206, 181-194 (2000)

53. Lorthois, S., Lagrée, P.-Y., Marc-Verges, J.-P., Cassot, F.: Maximal wall shear stress in arterial stenoses. Application to the internal carotid arteries. ASME J. Biomech. Eng. 122, 661-666 (2000)

54. Kassab, G.S., Schatz, A., Imoto, K., Fung, Y.C.: Remodelling of the bifurcation asymmetry of right coronary ventricular branches in hypertrophy. Ann. Biomed. Eng. 28, 424-430 (2000)

55. Zhai, Y., Brunskill, C.T., Lieber, B.B.: Inspiratory and expiratory steady flow analysis in a model symmetrically bifurcating airway. Trans. ASME J. Biomech. Eng. 119, 52-65 (1997)

56. Salsac, A.-V., Sparks, S.R., Chomaz, J-M., Lasheras, J.C.: Evolution of the wall shear stresses during the progressive enlargement of symmetric abdominal aortic aneurysm. J. Fluid Mech. 560, 19-51 (2006)

57. Alastruey, J., Parker, K.H., Peiró, J., Sherwin, S.J.: Analysing the pattern of pulse waves in arterial networks: a time-domain study. J. Eng. Math. 64(4), 331 (2009)

58. Smith, F.T., Badr, H., Dennis, S.C.R., Bates, S.: Numerical and asymptotic solutions for merging flow through a channel with an upstream splitter plate. J. Fluid Mech. 156, 63-81 (1985)

59. Smith, F.T., Jones, M.A.: One-to-few and one-to-many branching tube flows. J. Fluid Mech. 423, 1-31 (2000)

60. Bowles, R.I., Dennis, S.C.R., Purvis, R., Smith, F.T.: Multi-branching flows from one mother tube to many daughters or to a network. Phil. Trans. Roy. Soc. A 363, 1045-1055 (2005)

61. Smith, F.T., Ovenden, N.C., Franke, P.T., Doorly, D.J.: What happens to pressure when a flow enters a side branch? J. Fluid Mech. 479, 231-258 (2003)

62. Hall, P.: On the non-linear evolution of Gortler vortices in non-parallel boundary layers. IMA J. Appl. Math. 29, 173-196 (1982)

63. Smith, F.T., Tadjfar, M.: Direct simulations and modelling of basic three-dimensional bifurcating tube flows. J. Fluid Mech. 519, 1-32 (2004). Cambridge University Press

64. Pries, A.R., Secomb, T.W., Gaehtgens, P.: Structural adaptation and stability of microvascular networks: theory and simulations. Am. J. Physiol. 275, H347-H360 (1998)

65. Waters, S.L., Alastruey, J., Beard, D.A., Bovendeerd, P.H.M., Davies, P.F., Jayaraman, G., Jensen, O.E., Lee, J., Parker, K.H., Popel, A.S., Secomb, T.W., Sherwin, S.J., Shipley, R.J., Smith, N.P., van de Vosse, F.N.: Theoretical models for coronary vascular biomechanics; progress and challenges. Prog. Biophys. Mol. Biol. 104, 49-76 (2011)

66. Alarcon, T., Byrne, H.M., Maini, P.K.: A design principle for vascular beds: the effects of complex blood rheology. Microvascular Res. 69, 156-172 (2005)

67. Tutty, O.R.: Flow in a tube with a small side branch. J. Fluid Mech. 191, 79-109 (1988)

68. Sobey, I.J.: Introduction to Interactive Boundary Layer Theory. OUP (2001)

69. Rosenhead, L. (ed.): Laminar Boundary Layers. OUP (1963)

70. Prandtl, L. Über Flüssigkeitsbewegung bei sehr kleiner Reibung. Verh. III. Intern. Math. Kongr., Heidelberg, 1904, S. 484-491, Teubner, Leipzig, 1905. In English: On the motion of fluids of very small viscosity. NACA Tech Rept Server NACA Tech Memo 452 (1904)

71. Landau, L.D., Lifschitz, E.M.: Fluid Mechanics. Reprinted 2000, 2nd edn (2000)

72. Brown, S.N., Stewartson, K.: Laminar separation. Ann. Rev. Fluid Mech. 1, 45 (1969)

73. Van Dyke, M.: Perturbation Methods in Fluid Mechanics. Academic Press (1964) 
74. Schlichting, H., Gersten, K.: Boundary-Layer Theory. 8th revised and enlarged edition. Springer, Berlin (2000)

75. Kevorkian, J., Cole, J.D.: Perturbation Methods in Applied Mathematics, 2nd edn. Springer, Berlin (1981)

76. Batchelor, G.K.: An Introduction to Fluid Dynamics. CUP (1967)

77. Smith, F.T.: On the high Reynolds number theory of laminar flows. IMA J. Appl. Math. 28, 207-281 (1982)

78. Smith, F.T., Brighton, P.W.M., Jackson, P.S., Hunt, J.C.R.: On boundary layer flow past two dimensional obstacles. J. Fluid Mech. 113, 123-152 (1981)

79. Smith, F.T.: Flow through symmetrically constricted tubes. J. Inst. Maths. Applics. 21, 145-156 (1978)

80. Tillett, J.P.K.: On the laminar flow in a free jet of liquid at high Reynolds numbers. J. Fluid Mech. 32, 273-292 (1968)

81. Sychev, V.V., Ruban, A.I., Sychev, V.V., Korolev, G.L., Maroko, E.V.: Asymptotic Theory of Separated Flows. CUP (1998)

82. Smith, F.T., Rothmayer, A.P.: Incompressible Triple-Deck Theory. CRC Handbook of Fluid Dynamics, Chapts. 23-25 (1998)

83. Smith, F.T.: The separating flow through a severely constricted symmetric tube. J. Fluid Mech. 90(4), 725-754 (1979)

84. Smith, F.T., Dennis, S.C.R.: Steady flow through a channel with a symmetrical constriction in the form of a step. Proc. R. Soc. A. 372, 393-414 (1980)

85. Brotherton-Ratcliffe, R.V.: Boundary layer effects in liquid layer flows. PhD thesis, University of London (1987)

86. Milne-Thomson, L.M.: Theoretical Hydrodynamics. Courier Dover Publications (1996)

87. Comer, J.K., Kleinstreuer, C., Zhang, Z.: Flow structures and particle deposition patterns in doublebifurcation airway models. Part 1: air flow fields. J. Fluids 435, 25-54 (2001)

88. Smith, F.T., Jones, M.A.: AVM modelling by multi-branching tube flow: large flow rates and dual solutions. Math. Med. Biol. 20, 183-204 (2003)

89. Smith, F.T., Ovenden, N.C., Wu, G.X.: The effects of nonsymmetry in a branching flow network. J. Eng. Math. 63, 213-239 (2009)

90. Smith, F.T., Ovenden, N., Purvis, R.: Industrial and biomedical applications. In: Meier, G.E.A., Sreenivasan, K.R. (eds.) IUTAM Symposium on One Hundred Years of Boundary Layer Research. Solid Mechanics and its Applications, vol. 129, pp. 291-300. Springer, The Netherlands (2006)

91. White, A.H., Smith, F.T.: Wall-shape effects on multiphase flow in channels. Theor. Comput. Fluid Dyn. 26, 339-360 (2011)

92. Duck, P.W., Burggraf, O.R.: Spectral solutions for three-dimensional triple-deck flow over surface topography. J. Fluid Mech. 162, 1-22 (1986)

93. Smith, F.T., Bowles, R.I., Ovenden, N.C.: Multi-branching three-dimensional flow with substantial changes in vessel shapes. J. Fluid Mech. 614, 329-354 (2008). Cambridge University Press

94. Ovenden, N.C., Smith, F.T.: Three-dimensional branching flow analysis and computation. J. Fluid Mech. (2012, in preparation)

95. Smith, F.T.: Steady motion through a branching tube. Proc. Roy. Soc. A 355, 167-187 (1977)

96. Smith, F.T., Bowles, R.I., Davies, C.: On the spiking stages in deep transition and unsteady separation. J. Eng. Math. 45, 227-245 (2003) 